\title{
One-Pot Asymmetric Synthesis of Alkylidene 1-Alkylindan-1-ols Using Brønsted Acid and Palladium Catalysis
}

\author{
Réka J. Faggyas, Ewen D. D. Calder, Claire Wilson, and Andrew Sutherland*(-) \\ WestCHEM, School of Chemistry, University of Glasgow, The Joseph Black Building, Glasgow G12 8QQ United Kingdom
}

Supporting Information

ABSTRACT: A one-pot catalytic enantioselective allylboration/Mizoroki-Heck reaction of 2-bromoaryl ketones has been developed for the asymmetric synthesis of 3-methyleneindanes bearing a tertiary alcohol center. Brønsted acidcatalyzed allylboration with a chiral BINOL derivative was followed by a palladium-catalyzed Mizoroki-Heck cyclization, resulting in selective formation of the exo-alkene. This novel protocol provides a concise and scalable approach to 1-alkyl-3methyleneindan-1-ols in high enantiomeric ratios (up to 96:4 er). The potential of these compounds as chiral building blocks was demonstrated with efficient transformation to optically active diol and amino alcohol scaffolds.

\section{INTRODUCTION}

Chiral indan-1-ols are an important structural motif, ${ }^{1}$ used as key intermediates for the synthesis of biologically active substances, including anistatin $[\mathbf{1}$ (Figure 1$)],{ }^{2}$ a sesquiterpene

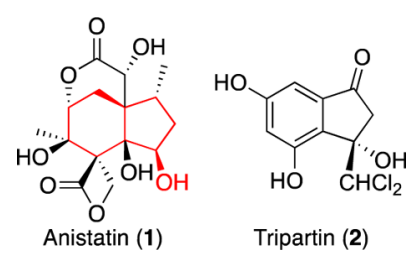

Figure 1. Structures of anistatin (1) and tripartin (2).

toxin from the seeds of the Japanese star anise, and dopamine reuptake blockers for the treatment of cocaine abuse. ${ }^{3}$ Chiral indan-1-ols are also found as core components of natural products, such as tripartin (2), a demethylase inhibitor, isolated from a Streptomyces species associated with dung beetle larva. ${ }^{4}$

Because of their importance, a number of approaches have been developed for the preparation of chiral indan-1-ol derivatives. ${ }^{1}$ Recent efforts have focused on the synthesis of optically active alkylidene indan-1-ols bearing a secondary alcohol center. These have been prepared from benzaldehyde derivatives using asymmetric allylation reactions followed by a metal-catalyzed $\mathrm{C}-\mathrm{C}$ bond forming reaction. ${ }^{5}$ One-pot methods have also been reported. For example, the groups of Schmalz ${ }^{6}$ and Fukuzawa ${ }^{7}$ described a one-pot asymmetric synthesis of 3-methyleneindan-1-ols from $o$-formylaryl iodides and triflates by sequential Sakurai-type allylstannylation and Mizoroki-Heck reaction in the presence of chiral $P, P$-ligands (e.g., Scheme 1a). More recently, we reported the one-pot synthesis of optically active 3-methyleneindan-1-ols from benzaldehydes using allylboration, catalyzed by a chiral binaphthyl-derived phosphoric acid, followed by a MizorokiHeck reaction (Scheme $1 b){ }^{8}$ This process was found to be general for both electron-deficient and electron-rich benzaldehydes, giving 3-methyleneindan-1-ols in excellent yields and high enantioselectivity.

While there are numerous methods for the synthesis of 3methyleneindan-1-ols from benzaldehydes, the more challenging analogous synthesis of 3-methyleneindan-1-ols with a tertiary alcohol center is less well-known. ${ }^{9}$ Only two general methods for the racemic synthesis of these compounds have been reported. A multistep approach has been described involving the preparation of allyl-substituted tertiary bromobenzyl alcohols, followed by a Mizoroki-Heck reaction, ${ }^{10}$ while Grigg and co-workers developed a one-pot synthesis of tertiarysubstituted 3-methyleneindan-1-ols by the palladium-catalyzed reaction of 2-bromoaryl ketones with allene. ${ }^{11}$ There is only one example of an asymmetric synthesis of a 1-alkyl-3methyleneindan-1-ol, described by Oestreich and co-workers, who used a desymmetrizing intramolecular Mizoroki-Heck reaction of an $o$-triflate-derived diallylated benzyl alcohol (Scheme 1c). ${ }^{12,13}$ This gave the tertiary-substituted 3methyleneindan-1-ol in 35\% yield and $46 \%$ ee.

Because of a lack of methods for accessing optically active 1alkyl-3-methyleneindan-1-ols, we were interested in developing a general and concise approach for the synthesis of these compounds from readily available 2-bromoaryl ketones. Herein, we now report the one-pot synthesis of 1-alkyl-3-methyleneindan-1-ols from 2-bromoaryl ketones using a combination of an asymmetric allylboration, followed by a Mizoroki-Heck reaction (Scheme 1d). The ease of synthesis has also allowed the investigation of these compounds as synthetic intermediates

Received: September 11, 2017

Published: October 6, 2017 
Scheme 1. Methods for the Asymmetric Synthesis of 3-Methyleneindan-1-ols

a) One-Pot Asymmetric Allylstannylation and Mizoroki-Heck Reaction

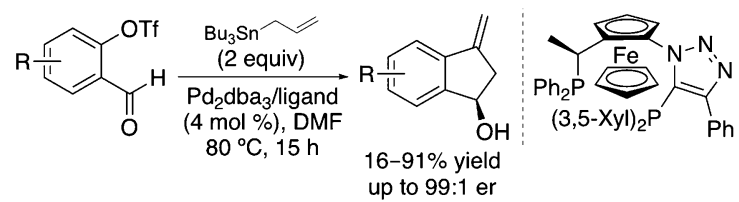

b) One-Pot Asymmetric Allylboration and Mizoroki-Heck Reaction

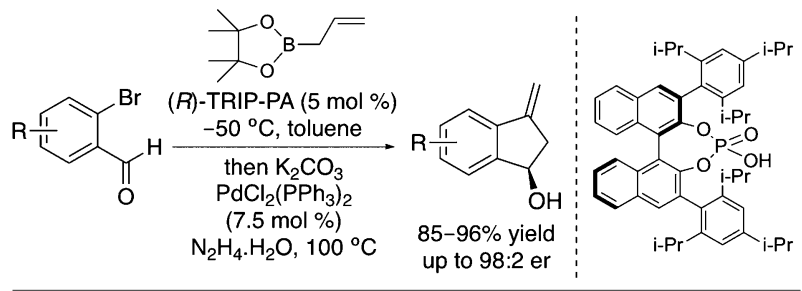

c) Desymmetrizing Intramolecular Mizoroki-Heck Reaction
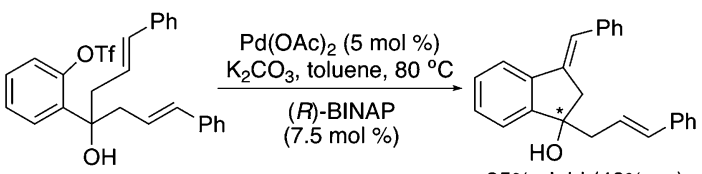

$35 \%$ yield $(46 \%$ ee $)$

d) This Work: One-Pot Asymmetric Synthesis of Alkylidene 1-Alkylindan-1-ols

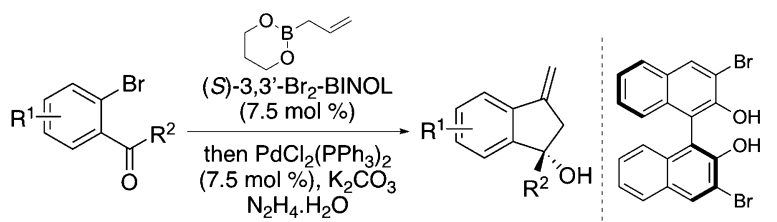

for the preparation of more functionalized diol and amino alcohol-derived Indane scaffolds.

\section{RESULTS AND DISCUSSION}

There are relatively few methods for the efficient catalytic asymmetric allylation of aryl ketones, ${ }^{14-19}$ and thus, we began our initial studies by screening various allylboronic esters and chiral Brønsted acids that would allow the effective allylation of 2-bromoaryl ketones. Crucially, conditions that would be compatible with a Mizoroki-Heck reaction were required. As we had previously shown that chiral binaphthyl-derived phosphoric acids could promote asymmetric allylation of 2bromobenzaldehydes, ${ }^{8}$ these were initially investigated for the catalytic allylation of 2-bromoacetophenone (3a) using allylboronic acid pinacol ester (5) (Table 1). However, neither unsubstituted phosphoric acid 7 nor sterically congested $(R)$ TRIP-PA $8^{20}$ promoted allylation (entries 1 and 2). 3,3'Dibrominated (S)-BINOL catalyst 9 , developed by Schaus and co-workers for asymmetric allylation of aryl ketones, was next investigated. ${ }^{15 \mathrm{~b}, \mathrm{c}}$ While allylation with 5 showed no reaction (entry 3), a $21 \%$ yield was obtained using allyldioxaborinane 6 and $t-\mathrm{BuOH}$ as the solvent (entry 4). To achieve the same result in toluene (required for the one-pot process), an increase in catalyst loading of 9 to $7.5 \mathrm{~mol} \%$ was required (entry 5). Further optimization, involving longer reaction times and an increase in temperature, eventually yielded the allylated product in quantitative yield (entry 7). Analysis of $\mathbf{4}$ by chiral HPLC indicated an enantiomeric ratio (er) of 98:2.

Conditions were then investigated for the one-pot, two-step synthesis of (1S)-2,3-dihydro-1-methyl-3-(methylene)indan-1ol (S-10a) from 2-bromoacetophenone (3a) using the (S)-3,3'-
Table 1. Asymmetric Allylation of 2-Bromoacetophenone (3a)

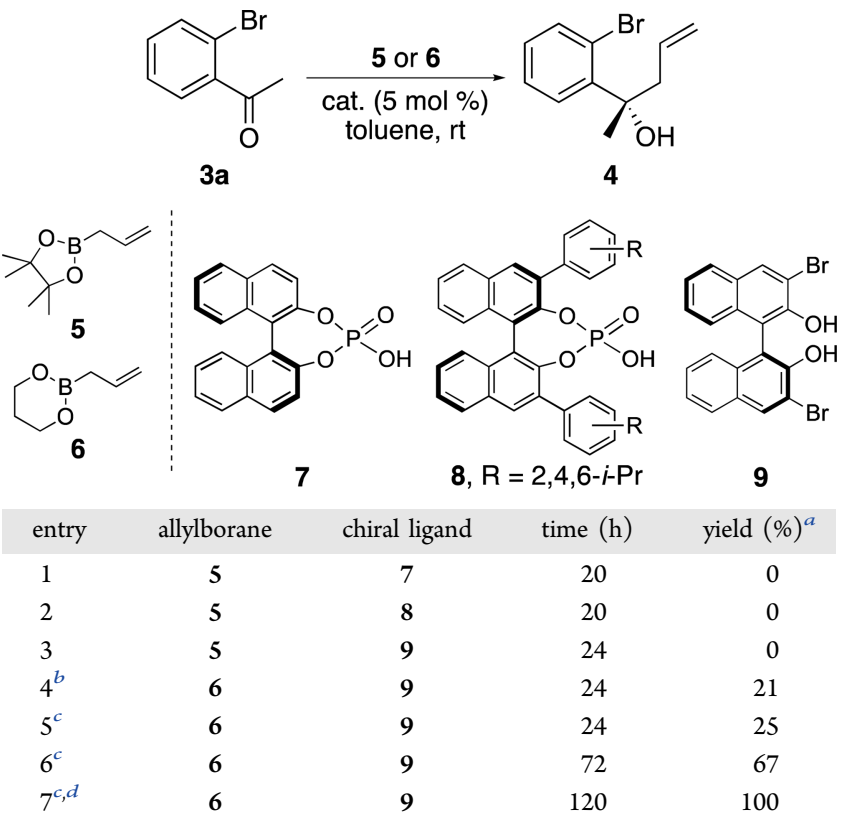

${ }^{a}$ Isolated yield of $4 .{ }^{b} t-\mathrm{BuOH}$ was used as the solvent. ${ }^{c} \mathrm{Here}, 7.5 \mathrm{~mol}$ $\% 9$ was used. ${ }^{d}$ The reaction temperature was increased to $35^{\circ} \mathrm{C}$.

$\mathrm{Br}_{2}$-BINOL-catalyzed allylation, followed by a Mizoroki-Heck reaction (Table 2). The first attempt involved the use of

Table 2. Optimization of the One-Pot Procedure

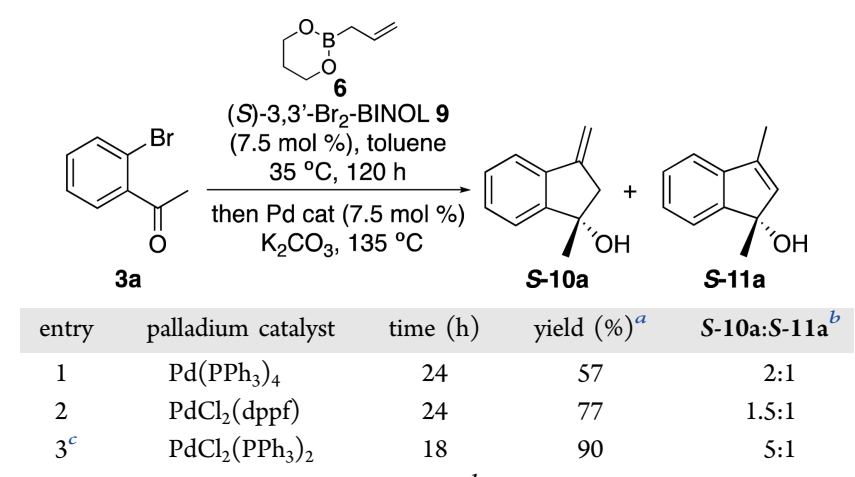

${ }^{a}$ Combined yield of $S$-10a and $S$-11a. ${ }^{b}$ Ratio determined by analysis of the ${ }^{1} \mathrm{H}$ NMR spectrum of the crude reaction mixture. ${ }^{c} \mathrm{~N}_{2} \mathrm{H}_{4} \cdot \mathrm{H}_{2} \mathrm{O}(0.4$ equiv) was added with a Pd catalyst.

$\mathrm{Pd}\left(\mathrm{PPh}_{3}\right)_{4}$ to catalyze the Mizoroki-Heck reaction. While this gave indanol $S-10$ a as the major product, endo-alkene isomer $S$ 11a was also formed (entry 1). ${ }^{21}$ An improvement in the overall yield of the one-pot, two-step process was achieved using $\mathrm{PdCl}_{2}(\mathrm{dppf})$; however, this resulted in a lower ratio of $\boldsymbol{S}$ 10a to $S$-11a (entry 2). The most selective catalyst was found to be $\mathrm{PdCl}_{2}\left(\mathrm{PPh}_{3}\right)_{2}$ (entry 3). In combination with the reductant hydrazine hydrate, this led to a 5:1 S-10a:S-11a ratio in $90 \%$ overall yield. Analysis of the products by chiral HPLC showed a 96:4 er. Purification by column chromatography allowed the isolation of $S-10$ a in $75 \%$ overall yield.

Following optimization of the one-pot process for the preparation of indanol $S-\mathbf{1 0 a}$, we explored the scope of the onepot method with a range of 2-bromoaryl ketones. These were prepared in a straightforward manner from commercially available 2-bromobenzaldehydes (Scheme 2). Addition of a 
Scheme 2. Synthesis of 2-Bromoaryl Ketones ${ }^{a}$

$$
\begin{aligned}
& \text { 3a } \\
& \begin{array}{l}
\mathrm{KNO}_{3}, \mathrm{H}_{2} \mathrm{SO}_{4} \\
0^{\circ} \mathrm{C} \text { to } \mathrm{rt}, 1.5 \mathrm{~h}
\end{array}
\end{aligned}
$$

${ }^{a}$ Isolated yields are shown. ${ }^{b}$ PDC was used to prepare ketone $3 \mathbf{h}$.

Grignard reagent gave the corresponding secondary alcohols that were then oxidized to the 2-bromoaryl ketones using either manganese oxide or a PDC/silica mixture. This allowed the scalable synthesis of a wide range of 2-bromoaryl ketones in good yields over the two steps. An additional substrate bearing a 5-nitro group ( $3 \mathrm{~g}$ ) was prepared in $54 \%$ yield by direct nitration of 2-bromoacetophenone $(3 \mathbf{a}) .^{22}$

Having produced a series of 2-bromoaryl ketones, we then applied these to the one-pot allylboration and Mizoroki-Heck process (Scheme 3). Initially, we wanted to demonstrate that either indanol enantiomer could be prepared using either $(S)$ or $(R)-3,3^{\prime}-\mathrm{Br}_{2}$-BINOL. Therefore, the one-pot process with 2 bromoacetophenone (3a) was repeated using (R) $-3,3^{\prime}-\mathrm{Br}_{2}-$ BINOL. As expected, this gave similar results, with indanol $R$ 10a produced with a $96: 4$ er and isolated in $70 \%$ overall yield. 2-Bromoaryl methyl ketones with various aryl substituents were then subjected to the one-pot process using $(S)-3,3^{\prime}-\mathrm{Br}_{2}$ BINOL. Substrates with electron-rich aryl substituents gave the two isomeric indanols in high yields over the two steps, with the major (1S)-3-methyleneindanols $10 \mathbf{a}-\mathbf{c}$ produced with a high er (from 89.5:10.5 to 96:4) and easily isolated in good yields $(61-75 \%)$. The exception to these general results was observed for methoxy-derived indanols 10d and 11d. While (1S)-3-methyleneindanol 10d was formed with a high er and isolated in a reasonable $47 \%$ overall yield, a modest 2:1 ratio of alkene isomers was generated from the one-pot process. In this case, we believe the methoxy substituent facilitates isomerization in the presence of the $\operatorname{Pd}(0)$ catalyst following completion of the Mizoroki-Heck reaction. It should be noted that other studies that have prepared electron-rich, racemic 1-alkyl-3-methyleneindan-1-ols by a single-step Mizoroki-Heck reaction have been complicated by the formation of an indene byproduct, formed via dehydration. ${ }^{10}$ In this study, using the asymmetric one-pot process, no dehydration was observed with analogous substrates (e.g., 3c). Electron-deficient 2-bromoaryl ketones were then investigated and also found to be substrates for this process. While halogenated analogues 10e and 10 were formed with a high er and isolated in good overall yields, nitro-substituted (1S)-3-methyleneindanol $10 \mathrm{~g}$ was formed in only $19 \%$ yield, demonstrating a limitation of this approach. Interestingly, pyridine analogue $11 \mathrm{~h}$ was isolated solely as the endo-alkene isomer, in $63 \%$ yield. In this case, isomerization of the exo-alkene is likely facilitated by
Scheme 3. Scope of the One-Pot Two-Step Process ${ }^{a}$

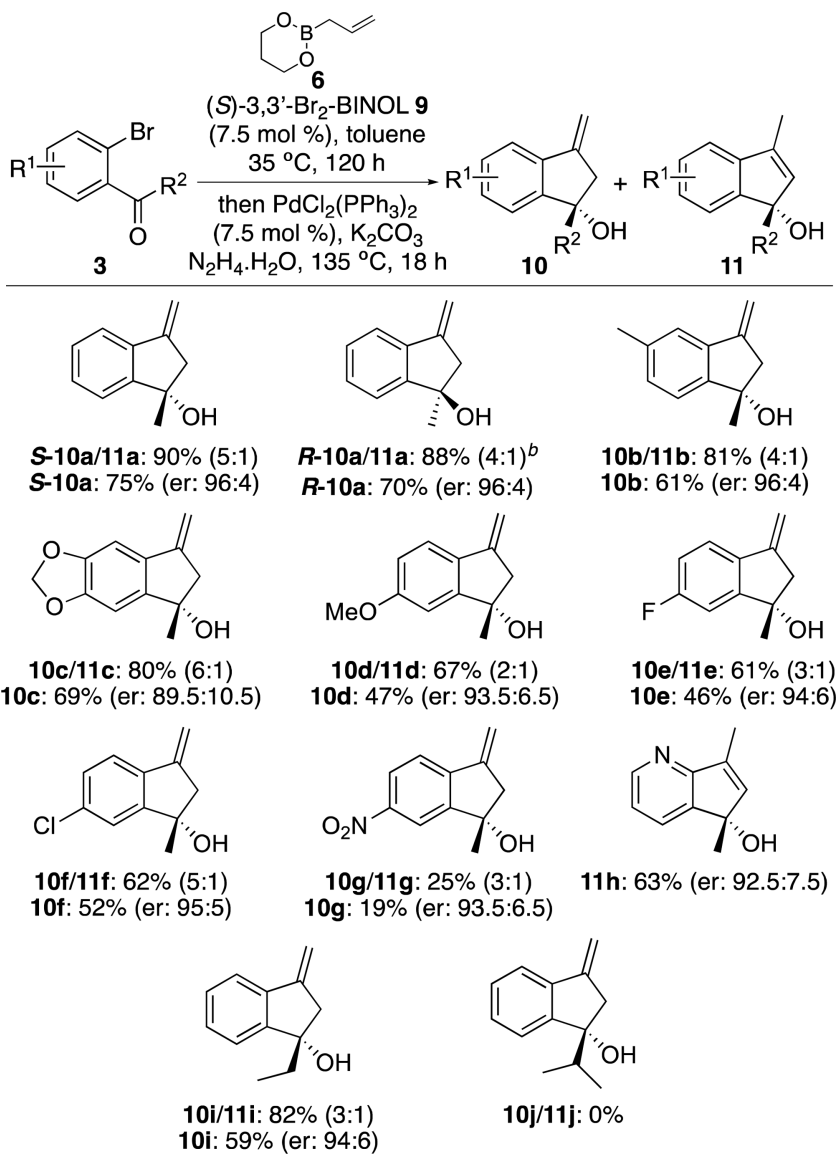

${ }^{a}$ Ratio of isomers 10 and $\mathbf{1 1}$ determined by analysis of the ${ }^{1} \mathrm{H}$ NMR spectrum of the crude reaction mixture. Enantiomeric ratio determined by chiral HPLC. ${ }^{b}$ The one-pot process was performed using (R)-3,3'$\mathrm{Br}_{2}$-BINOL.

coordination of the $\operatorname{Pd}(0)$ catalyst with the adjacent nitrogen atom. This study also briefly investigated the scope of the ketone side chain. Use of the one-pot process with 2-bromoaryl ethyl ketone $3 \mathbf{i}$ gave a high overall yield of the indanol products with (1S)-3-methyleneindanol 10i produced with a 94:6 er and isolated in 59\% yield. However, increasing the size of the side chain with an isopropyl group $(3 \mathbf{j})$ completely inhibited the allylation step. While $(S)-3,3^{\prime}-\mathrm{Br}_{2}-\mathrm{BINOL}$-catalyzed allylations have been performed with a range of alkyl-substituted aryl ketones, ${ }^{15 b, c}$ compounds with both a bulky alkyl group and a 2bromoaryl substituent are not substrates for this reaction.

In the next stage of this project, we wanted to demonstrate that the optically active 1-alkyl-3-methyleneindanols that can now be accessed using our concise one-pot approach could be further functionalized to generate chiral synthetic building blocks. In a preliminary study, we have shown that the methylene unit of the indanols can be used to incorporate a second stereogenic center through an oxidation and reduction sequence (Scheme 4). Initially, multigram quantities of $\boldsymbol{S}$-10a were produced by scale-up of the one-pot process. During these reactions, it was found that the catalyst loading of both $(S)-3,3^{\prime}$ $\mathrm{Br}_{2}$-BINOL 9 and $\mathrm{PdCl}_{2}\left(\mathrm{PPh}_{3}\right)_{2}$ could be decreased to $5 \mathrm{~mol}$ $\%$, while still maintaining the er (96:4) and high yield (72\%) of $\boldsymbol{S}$-10a. Ozonolysis of $\boldsymbol{S}$-10a under standard conditions gave indanone 14 in $81 \%$ yield. Indanone 14 is a structural analogue of tripartin [2 (Figure 1)], a natural product from bacteria 
Scheme 4. Synthesis of 3-Hydroxy- and 3-Aminoindanols ${ }^{a}$

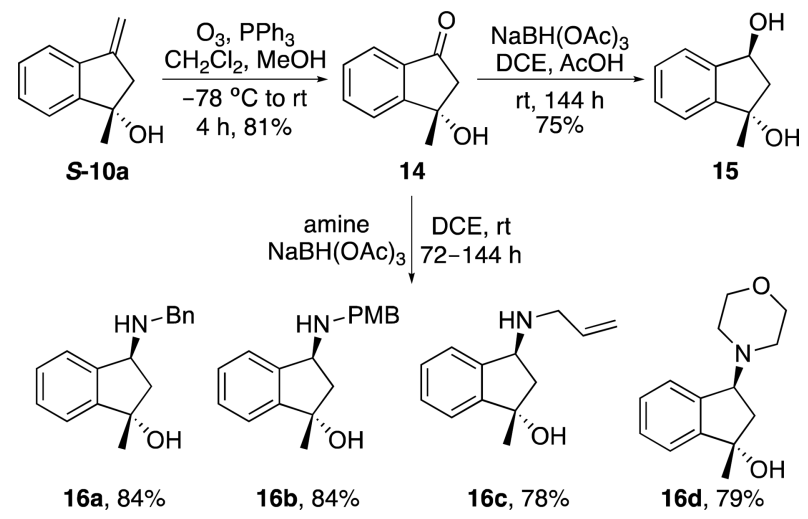

${ }^{a}$ Isolated yields are shown.

associated with the dung beetle Copris tripartitus. ${ }^{4}$ Various borohydride reagents were next investigated for reduction of 14. The most selective was sodium triacetoxyborohydride, and while the use of this required a prolonged reaction time, diol $\mathbf{1 5}$ was isolated as a single diastereomer in $75 \%$ yield. The highly selective nature of sodium triacetoxyborohydride was used for reductive amination of indanone $\mathbf{1 4}$. With various amines, this gave corresponding 3-aminoindanols $16 \mathbf{a}-\mathbf{d}$ as single diastereomers in high yields $(78-84 \%)$. It was proposed that reduction of indanone 14 and the corresponding imines was achieved via a directed mechanism, involving coordination of the reducing agent to the 1-hydroxyl group, followed by reduction of the same face of the ketone/imine. This was confirmed by X-ray crystallographic analysis of diol 15 and morpholine derivative 16d, which clearly show the anti relationship of the C-1 hydroxyl and C-3 hydroxyl/amine groups. $^{23}$

\section{CONCLUSIONS}

In summary, a one-pot asymmetric allylboration and MizorokiHeck reaction of 2-bromoaryl ketones has been developed for the concise synthesis of optically active 1-alkyl-3-methyleneindanes bearing a tertiary alcohol center. As well as generating these compounds with high enantiomeric ratios, the method is scalable and can be used to access either enantiomer. Now that a general procedure for the preparation of these compounds has been established, the synthetic utility of these highly functional scaffolds can be realized. We have shown that these compounds can be readily converted to hydroxyl and amine chiral building blocks in a highly diastereoselective manner. Investigation of further applications of these chiral 3methyleneindanes is currently underway.

\section{EXPERIMENTAL SECTION}

All reagents and starting materials were obtained from commercial sources and used as received. All dry solvents were purified using a solvent purification system. All reactions were performed in oven-dried glassware under an atmosphere of argon unless otherwise stated. Brine refers to a saturated solution of sodium chloride. Flash column chromatography was performed using silica gel 60 $(40-63 \mu \mathrm{m})$. Aluminum-backed plates precoated with silica gel $60 \mathrm{~F}_{254}$ were used for thin layer chromatography and were visualized with a UV lamp or by staining with potassium permanganate. ${ }^{1} \mathrm{H}$ NMR spectra were recorded on a NMR spectrometer at either 400 or $500 \mathrm{MHz}$, and data are reported as follows: chemical shift in parts per million relative to tetramethylsilane or the solvent as the internal standard $\left(\mathrm{CDCl}_{3}, \delta\right.$
7.26, or $\mathrm{CD}_{3} \mathrm{OD}, \delta 3.31$ ), multiplicity (s, singlet; d, doublet; t, triplet; $\mathrm{q}$, quartet; $\mathrm{m}$, multiplet or overlap of nonequivalent resonances, integration). ${ }^{13} \mathrm{C}$ NMR spectra were recorded on a NMR spectrometer at either 101 or $126 \mathrm{MHz}$, and data are reported as follows: chemical shift in parts per million relative to tetramethylsilane or the solvent as the internal standard $\left(\mathrm{CDCl}_{3}, \delta 77.0\right.$, or $\left.\mathrm{CD}_{3} \mathrm{OD}, \delta 49.0\right)$, multiplicity with respect to hydrogen (deduced from DEPT experiments, $\mathrm{C}, \mathrm{CH}$, $\mathrm{CH}_{2}$, or $\mathrm{CH}_{3}$ ). IR spectra were recorded on a FTIR spectrometer; wavenumbers are indicated in inverse centimeters. Mass spectra were recorded using electron impact, chemical ionization, or electrospray techniques. HRMS spectra were recorded using a dual-focusing magnetic analyzer mass spectrometer. Melting points are uncorrected. Optical rotations were determined as solutions irradiating with the sodium $\mathrm{D}$ line $(\lambda=589 \mathrm{~nm})$ using a polarimeter. $[\alpha]_{\mathrm{D}}$ values are given in units of $10^{-1}$ degree square centimeter per gram. Chiral HPLC methods were calibrated with the corresponding racemic mixtures.

General Procedure for the Synthesis of Secondary Alcohols (13). To a stirred solution of a 2-bromobenzaldehyde (1 equiv) in dry diethyl ether $(2 \mathrm{~mL} / \mathrm{mmol})$ at $-78{ }^{\circ} \mathrm{C}$ under an argon atmosphere was added dropwise a solution of methylmagnesium bromide ( $1 \mathrm{M}$ in dibutyl ether, 2 equiv), ethylmagnesium bromide ( $1 \mathrm{M}$ in THF, 2 equiv), or isopropylmagnesium bromide ( $1 \mathrm{M}$ in THF, 2 equiv). After addition, the reaction mixture was allowed to warm to $0{ }^{\circ} \mathrm{C}$ and stirred for $3 \mathrm{~h}$. The reaction was then quenched with a saturated solution of aqueous ammonium chloride $(10-15 \mathrm{~mL} / \mathrm{mmol})$. The product was extracted using diethyl ether $(3 \times 20 \mathrm{~mL} / \mathrm{mmol})$. The combined organic layers were washed with brine $(2 \times 20 \mathrm{~mL} / \mathrm{mmol})$, dried over $\mathrm{MgSO}_{4}$, filtered, and concentrated in vacuo. Purification of the crude material using silica gel flash column chromatography eluting with diethyl ether in petroleum ether gave the secondary alcohols.

1-(2'-Bromo-4'-methylphenyl)ethan-1-ol (13b). The reaction was performed according to the general procedure using 2-bromo-4methylbenzaldehyde (12b) (0.40 g, $2.0 \mathrm{mmol})$ and a methylmagnesium bromide solution $(4.0 \mathrm{~mL}, 4.0 \mathrm{mmol})$. Purification by flash column chromatography eluting with $10 \%$ diethyl ether in petroleum ether (40-60) gave 1-(2'-bromo-4'-methylphenyl)ethan-1-ol (13b) $(0.42 \mathrm{~g}, 98 \%)$ as a colorless oil: IR (neat) $3335,2974,1609,1488$ $\mathrm{cm}^{-1}$; ${ }^{1} \mathrm{H} \mathrm{NMR}\left(400 \mathrm{MHz}, \mathrm{CDCl}_{3}\right) \delta 1.47(\mathrm{~d}, J=6.5 \mathrm{~Hz}, 3 \mathrm{H}), 1.95$ $(\mathrm{d}, J=3.5 \mathrm{~Hz}, 1 \mathrm{H}), 2.31(\mathrm{~s}, 3 \mathrm{H}), 5.21(\mathrm{qd}, J=6.5,3.5 \mathrm{~Hz}, 1 \mathrm{H}), 7.15$ (br d, $J=8.0 \mathrm{~Hz}, 1 \mathrm{H}), 7.35(\mathrm{br} \mathrm{d}, J=0.9 \mathrm{~Hz}, 1 \mathrm{H}), 7.46(\mathrm{~d}, J=8.0 \mathrm{~Hz}$, $1 \mathrm{H}) ;{ }^{13} \mathrm{C}$ NMR $\left(101 \mathrm{MHz}, \mathrm{CDCl}_{3}\right) \delta 20.7\left(\mathrm{CH}_{3}\right), 23.6\left(\mathrm{CH}_{3}\right), 69.0$ $(\mathrm{CH}), 121.6(\mathrm{C}), 126.4(\mathrm{CH}), 128.7(\mathrm{CH}), 133.1(\mathrm{CH}), 138.9(\mathrm{C})$, 141.5 (C); MS (ESI) $m / z 237\left(\mathrm{MNa}^{+}, 100\right)$; HRMS (ESI) calcd for $\mathrm{C}_{9} \mathrm{H}_{11}{ }^{79} \mathrm{BrNaO}\left(\mathrm{MNa}^{+}\right) 236.9885$, found 236.9882 .

1-(2'-Bromo-4',5'-methylenedioxyphenyl)ethan-1-ol (13c). ${ }^{24}$ The reaction was performed according to the general procedure using 2-bromo-4,5-methylenedioxybenzaldehyde (12c) $(0.69 \mathrm{~g}, 3.0 \mathrm{mmol})$ and a methylmagnesium bromide solution $(6.0$ $\mathrm{mL}, 6.0 \mathrm{mmol})$. Purification by flash column chromatography eluting with $10 \%$ diethyl ether in petroleum ether $(40-60)$ gave 1 - $\left(2^{\prime}\right.$-bromo$4^{\prime}, 5^{\prime}$-methylenedioxyphenyl)ethan-1-ol (13c) $(0.63 \mathrm{~g}, 86 \%)$ as a colorless oil that solidified upon standing to give a white solid: $\mathrm{mp} 51-$ $53{ }^{\circ} \mathrm{C}\left(\right.$ lit. $\left.^{24} 52-53{ }^{\circ} \mathrm{C}\right) ;{ }^{1} \mathrm{H}$ NMR $\left(400 \mathrm{MHz}, \mathrm{CDCl}_{3}\right) \delta 1.43(\mathrm{~d}, J=$ $6.4 \mathrm{~Hz}, 3 \mathrm{H}), 1.87(\mathrm{~d}, J=3.3 \mathrm{~Hz}, 1 \mathrm{H}), 5.18(\mathrm{qd}, J=6.4,3.3 \mathrm{~Hz}, 1 \mathrm{H})$, $5.97(\mathrm{~d}, J=1.6 \mathrm{~Hz}, 1 \mathrm{H}), 5.98(\mathrm{~d}, J=1.6 \mathrm{~Hz}, 1 \mathrm{H}), 6.96(\mathrm{~s}, 1 \mathrm{H}), 7.09$ $(\mathrm{s}, 1 \mathrm{H}) ;{ }^{13} \mathrm{C} \mathrm{NMR}\left(101 \mathrm{MHz}, \mathrm{CDCl}_{3}\right) \delta 23.6\left(\mathrm{CH}_{3}\right), 69.1(\mathrm{CH})$, $101.7\left(\mathrm{CH}_{2}\right), 106.6(\mathrm{CH}), 111.8(\mathrm{C}), 112.4(\mathrm{CH}), 138.1(\mathrm{C}), 147.4$ (C), 147.8 (C); MS (ESI) $m / z 269\left(\mathrm{MNa}^{+}, 100\right), 267$ (98).

1-(2'-Bromo-5'-methoxyphenyl)ethan-1-ol (13d). ${ }^{10}$ The reaction was performed according to the general procedure using 2bromo-5-methoxybenzaldehyde (12d) (0.44 g, $3.0 \mathrm{mmol})$ and a methylmagnesium bromide solution $(6.0 \mathrm{~mL}, 6.0 \mathrm{mmol})$. Purification by flash column chromatography eluting with $10 \%$ diethyl ether in petroleum ether $(40-60)$ gave 1 - $\left(2^{\prime}\right.$-bromo- 5 '-methoxyphenyl)ethan1-ol (13d) (0.63 g, 90\%) as a colorless oil. Spectroscopic data were consistent with the literature: ${ }^{10}{ }^{1} \mathrm{H} \mathrm{NMR}\left(400 \mathrm{MHz}, \mathrm{CDCl}_{3}\right) \delta 1.47$ $(\mathrm{d}, J=6.4 \mathrm{~Hz}, 3 \mathrm{H}), 1.96(\mathrm{~d}, J=3.4 \mathrm{~Hz}, 1 \mathrm{H}), 3.81(\mathrm{~s}, 3 \mathrm{H}), 5.19(\mathrm{qd}, J$ $=6.4,3.4 \mathrm{~Hz}, 1 \mathrm{H}), 6.69(\mathrm{dd}, J=8.8,3.1 \mathrm{~Hz}, 1 \mathrm{H}), 7.16(\mathrm{~d}, J=3.1 \mathrm{~Hz}$, $1 \mathrm{H}), 7.39(\mathrm{~d}, J=8.8 \mathrm{~Hz}, 1 \mathrm{H}) ;{ }^{13} \mathrm{C}$ NMR $\left(101 \mathrm{MHz}, \mathrm{CDCl}_{3}\right) \delta 23.6$ $\left(\mathrm{CH}_{3}\right), 55.5\left(\mathrm{CH}_{3}\right), 69.2(\mathrm{CH}), 111.8(\mathrm{C}), 112.0(\mathrm{CH}), 114.7(\mathrm{CH})$, 
133.2 (CH), 145.8 (C), 159.4 (C); MS (EI) $m / z 232\left(\mathrm{M}^{+}, 39\right), 230$ (40).

1-(2'-Bromo-5'-fluorophenyl)ethan-1-ol (13e). The reaction was performed according to the general procedure using 2-bromo-5fluorobenzaldehyde $(12 \mathrm{e})(0.61 \mathrm{~g}, 3.0 \mathrm{mmol})$ and a methylmagnesium bromide solution $(6.0 \mathrm{~mL}, 6.0 \mathrm{mmol})$. Purification by flash column chromatography eluting with $10 \%$ diethyl ether in petroleum ether (40-60) gave 1-(2'-bromo-5'-fluorophenyl)ethan-1-ol (13e) (0.58 g, $88 \%)$ as a colorless oil: IR (neat) $3323,2974,1580,1465 \mathrm{~cm}^{-1} ;{ }^{1} \mathrm{H}$ NMR $\left(400 \mathrm{MHz}, \mathrm{CDCl}_{3}\right) \delta 1.47(\mathrm{~d}, J=6.4 \mathrm{~Hz}, 3 \mathrm{H}), 2.05(\mathrm{~d}, J=3.5$ $\mathrm{Hz}, 1 \mathrm{H}$ ), 5.14-5.23 (m, 1H), 6.86 (ddd, $J=8.6,8.0,3.2 \mathrm{~Hz}, 1 \mathrm{H}$ ), 7.34 $(\mathrm{dd}, J=9.8,3.2 \mathrm{~Hz}, 1 \mathrm{H}), 7.46(\mathrm{dd}, J=8.6,5.2 \mathrm{~Hz}, 1 \mathrm{H}) ;{ }^{13} \mathrm{C} \mathrm{NMR}$ $\left(101 \mathrm{MHz}, \mathrm{CDCl}_{3}\right) \delta 23.5\left(\mathrm{CH}_{3}\right), 69.1(\mathrm{CH}), 114.0\left(\mathrm{~d},{ }^{2} J_{\mathrm{C}-\mathrm{F}}=24.0\right.$ $\mathrm{Hz}, \mathrm{CH}), 115.3(\mathrm{C}), 115.9\left(\mathrm{~d},{ }^{2} J_{\mathrm{C}-\mathrm{F}}=22.8 \mathrm{~Hz}, \mathrm{CH}\right), 133.8\left(\mathrm{~d},{ }^{3} J_{\mathrm{C}-\mathrm{F}}=\right.$ $7.8 \mathrm{~Hz}, \mathrm{CH}), 147.0\left(\mathrm{~d},{ }^{3} J_{\mathrm{C}-\mathrm{F}}=6.7 \mathrm{~Hz}, \mathrm{C}\right), 162.5\left(\mathrm{~d},{ }^{1} J_{\mathrm{C}-\mathrm{F}}=247.2 \mathrm{~Hz}\right.$, C); MS (EI) $m / z 218$ (M+ 38), 205 (82), 203 (100), 175 (16), 152 (38), 123 (11), 96 (60); HRMS (EI) calcd for $\mathrm{C}_{8} \mathrm{H}_{8}{ }^{79} \mathrm{BrFO}\left(\mathrm{M}^{+}\right)$ 217.9743, found 217.9731 .

1-(2'-Bromo-5'-chlorophenyl)ethan-1-ol (13f). The reaction was performed according to the general procedure using 2-bromo-5chlorobenzaldehyde (12f) $(0.66 \mathrm{~g}, 3.0 \mathrm{mmol})$ and a methylmagnesium bromide solution $(6.0 \mathrm{~mL}, 6.0 \mathrm{mmol})$. Purification by flash column chromatography eluting with $10 \%$ diethyl ether in petroleum ether (40-60) gave 1-(2'-bromo-5'-chlorophenyl)ethan-1-ol (13f) (0.68 g, $96 \%$ ) as a colorless oil: IR (neat) $3336,2976,1449 \mathrm{~cm}^{-1}$; ${ }^{1} \mathrm{H}$ NMR $\left(400 \mathrm{MHz}, \mathrm{CDCl}_{3}\right) \delta 1.47(\mathrm{~d}, J=6.4 \mathrm{~Hz}, 3 \mathrm{H}), 2.01(\mathrm{~d}, J=3.7 \mathrm{~Hz}$, $1 \mathrm{H}), 5.18(\mathrm{qd}, J=6.4,3.7 \mathrm{~Hz}, 1 \mathrm{H}), 7.11(\mathrm{dd}, J=8.4,2.6 \mathrm{~Hz}, 1 \mathrm{H}), 7.43$ $(\mathrm{d}, J=8.4 \mathrm{~Hz}, 1 \mathrm{H}), 7.60(\mathrm{~d}, J=2.6 \mathrm{~Hz}, 1 \mathrm{H}) ;{ }^{13} \mathrm{C} \mathrm{NMR}(101 \mathrm{MHz}$, $\left.\mathrm{CDCl}_{3}\right) \delta 23.6\left(\mathrm{CH}_{3}\right), 69.0(\mathrm{CH}), 119.2(\mathrm{C}), 127.0(\mathrm{CH}), 128.8$ $(\mathrm{CH}), 133.7(\mathrm{CH}), 134.1(\mathrm{C}), 146.5(\mathrm{C})$; MS (EI) $\mathrm{m} / \mathrm{z} 236\left(\mathrm{M}^{+}, 38\right)$, 234 (29), 223 (24), 221 (100), 219 (81), 112 (56), 75 (27); HRMS (EI) calcd for $\mathrm{C}_{8} \mathrm{H}_{8}{ }^{81} \mathrm{Br}^{35} \mathrm{ClO}\left(\mathrm{M}^{+}\right) 235.9425$, found 235.9430 .

1-(2'-Bromo-3'-pyridyl)ethan-1-ol (13h). The reaction was performed according to the general procedure using 2-bromopyridine-3-carboxaldehyde $(\mathbf{1 2 h})(1.00 \mathrm{~g}, 5.40 \mathrm{mmol})$ and a methylmagnesium bromide solution $(11.0 \mathrm{~mL}, 10.8 \mathrm{mmol})$. Purification by flash column chromatography eluting with $10 \%$ diethyl ether in petroleum ether (40-60) gave 1-( $2^{\prime}$-bromo-3'-pyridyl)ethan-1-ol (13h) $(0.94 \mathrm{~g}, 87 \%)$ as a yellow oil: IR (neat) 3410, 2932, 1651, $1389 \mathrm{~cm}^{-1}$; ${ }^{1} \mathrm{H}$ NMR $\left(400 \mathrm{MHz}, \mathrm{CDCl}_{3}\right) \delta 1.47(\mathrm{~d}, J=6.4 \mathrm{~Hz}, 3 \mathrm{H})$, $3.76($ br s, $1 \mathrm{H}), 5.12-5.20(\mathrm{~m}, 1 \mathrm{H}), 7.28(\mathrm{dd}, J=7.6,4.8 \mathrm{~Hz}, 1 \mathrm{H})$, $7.93(\mathrm{dd}, J=7.6,2.0 \mathrm{~Hz}, 1 \mathrm{H}), 8.16(\mathrm{dd}, J=4.8,2.0 \mathrm{~Hz}, 1 \mathrm{H}) ;{ }^{13} \mathrm{C}$ NMR $\left(101 \mathrm{MHz}, \mathrm{CDCl}_{3}\right) \delta 23.7\left(\mathrm{CH}_{3}\right), 67.9(\mathrm{CH}), 123.4(\mathrm{CH})$, $135.8(\mathrm{CH}), 140.9(\mathrm{C}), 142.6(\mathrm{C}), 148.4(\mathrm{CH})$; MS (ESI) $\mathrm{m} / \mathrm{z} 224$ $\left(\mathrm{MNa}^{+}, 98\right)$; HRMS (ESI) calcd for $\mathrm{C}_{7} \mathrm{H}_{8}{ }^{79} \mathrm{BrNNaO}\left(\mathrm{MNa}^{+}\right)$ 223.9681, found 223.9674.

1-(2'-Bromophenyl)propan-1-ol (13i). ${ }^{25}$ The reaction was performed according to the general procedure using 2-bromobenzaldehyde (12a) $(1.0 \mathrm{~mL}, 8.0 \mathrm{mmol})$ and an ethylmagnesium bromide solution $(16 \mathrm{~mL}, 16 \mathrm{mmol})$. Purification by flash column chromatography eluting with $10 \%$ diethyl ether in petroleum ether $(40-60)$ gave 1 -(2'-bromophenyl)propan-1-ol (13i) $(1.40 \mathrm{~g}, 81 \%)$ as a colorless oil. Spectroscopic data were consistent with the literature: ${ }^{25}$ ${ }^{1} \mathrm{H}$ NMR $\left(400 \mathrm{MHz}, \mathrm{CDCl}_{3}\right) \delta 1.01(\mathrm{td}, J=7.4,0.8 \mathrm{~Hz}, 3 \mathrm{H}), 1.65-$ $1.90(\mathrm{~m}, 2 \mathrm{H}), 1.95-2.05(\mathrm{~m}, 1 \mathrm{H}), 5.01(\mathrm{dt}, J=8.0,4.0 \mathrm{~Hz}, 1 \mathrm{H}), 7.12$ (br t, $J=7.5 \mathrm{~Hz}, 1 \mathrm{H}), 7.33$ (br t, $J=7.5 \mathrm{~Hz}, 1 \mathrm{H}), 7.49-7.56(\mathrm{~m}, 1 \mathrm{H})$; ${ }^{13} \mathrm{C}$ NMR $\left(101 \mathrm{MHz}, \mathrm{CDCl}_{3}\right) \delta 10.1\left(\mathrm{CH}_{3}\right), 30.6\left(\mathrm{CH}_{2}\right), 74.2(\mathrm{CH})$, $122.2(\mathrm{C}), 127.4(\mathrm{CH}), 127.6(\mathrm{CH}), 128.7(\mathrm{CH}), 132.6(\mathrm{CH}), 143.6$ (C); MS (ESI) $m / z 239\left(\mathrm{MNa}^{+}, 95\right), 237$ (100).

1-(2'-Bromophenyl)-2-methylpropan-1-ol (13j). ${ }^{26}$ The reaction was performed according to the general procedure using 2bromobenzaldehyde $(\mathbf{1 2 a})(1.0 \mathrm{~mL}, 8.0 \mathrm{mmol})$ and an isopropylmagnesium bromide solution $(16 \mathrm{~mL}, 16 \mathrm{mmol})$. Purification by flash column chromatography eluting with $10 \%$ diethyl ether in petroleum ether (40-60) gave 1-(2'-bromophenyl)-2-methylpropan-1-ol (13j) $(0.91 \mathrm{~g}, 57 \%)$ as a colorless oil. Spectroscopic data were consistent with the literature: ${ }^{26}{ }^{1} \mathrm{H}$ NMR $\left(400 \mathrm{MHz} \mathrm{CDCl}_{3}\right) \delta 0.95(\mathrm{~d}, J=6.8$ $\mathrm{Hz}, 3 \mathrm{H}), 0.97(\mathrm{~d}, J=6.8 \mathrm{~Hz}, 3 \mathrm{H}), 1.85(\mathrm{br} \mathrm{s}, 1 \mathrm{H}), 2.00-2.14(\mathrm{~m}, 1 \mathrm{H})$, $4.86(\mathrm{~d}, J=6.0 \mathrm{~Hz}, 1 \mathrm{H}), 7.09-7.15(\mathrm{~m}, 1 \mathrm{H}), 7.29-7.36(\mathrm{~m}, 1 \mathrm{H}), 7.50$ $(\mathrm{td}, J=7.6,1.6 \mathrm{~Hz}, 2 \mathrm{H}) ;{ }^{13} \mathrm{C} \mathrm{NMR}\left(101 \mathrm{MHz}, \mathrm{CDCl}_{3}\right) \delta 16.8\left(\mathrm{CH}_{3}\right)$,
$19.5\left(\mathrm{CH}_{3}\right), 34.0(\mathrm{CH}), 77.6(\mathrm{CH}), 122.7(\mathrm{C}), 127.4(\mathrm{CH}), 128.3$ $(\mathrm{CH}), 128.6(\mathrm{CH}), 132.6(\mathrm{CH}), 142.9(\mathrm{C})$; MS (EI) $\mathrm{m} / \mathrm{z} 230\left(\mathrm{M}^{+}\right.$, 8), 228 (10), 187 (81), 185 (100), 172 (17), 157 (9), 105 (9), 84 (23), 77 (43).

General Procedure A for Oxidation to 2-Bromoaryl Ketones (3). To a stirred solution of a secondary alcohol (1 equiv) in chloroform $(3 \mathrm{~mL} / \mathrm{mmol})$ at room temperature was added manganese(IV) oxide (10 equiv). The reaction mixture was stirred for $18 \mathrm{~h}$ under reflux. After cooling to room temperature, the mixture was filtered through a pad of Celite, which was washed with diethyl ether $(2 \times 15 \mathrm{~mL} / \mathrm{mmol})$. The filtrate was then concentrated. The crude product was purified using silica gel flash column chromatography, eluting with diethyl ether in petroleum ether to give the corresponding ketone derivatives.

General Procedure B for Oxidation to 2-Bromoaryl Ketones (3). To a stirred solution of a secondary alcohol ( 1 equiv) in dichloromethane $(5 \mathrm{~mL} / \mathrm{mmol})$ at room temperature was added a homogeneous mixture of PCC and silica gel (1:1 by mass, 3 equiv). The resulting suspension was stirred for $2 \mathrm{~h}$. The mixture was filtered through a pad of silica gel, which was washed with dichloromethane (2 $\times 30 \mathrm{~mL} / \mathrm{mmol}$ ). The filtrate was then concentrated. The crude product was purified using silica gel flash column chromatography, eluting with diethyl ether in petroleum ether to give the corresponding ketone derivatives.

1-(2'-Bromo-4'-methylphenyl)ethan-1-one (3b). ${ }^{27}$ The reaction was performed according to general procedure A using 1-(2'bromo-4'-methylphenyl)ethan-1-ol (13b) (0.42 g, $2.0 \mathrm{mmol})$. Purification by flash column chromatography eluting with $10 \%$ diethyl ether in petroleum ether $(40-60)$ gave 1 - $\left(2^{\prime}\right.$-bromo- $4^{\prime}$ methylphenyl)ethan-1-one (3b) (0.33 g, 79\%) as a yellow oil. Spectroscopic data were consistent with the literature: ${ }^{27}{ }^{1} \mathrm{H}$ NMR $\left(400 \mathrm{MHz}, \mathrm{CDCl}_{3}\right) \delta 2.36(\mathrm{~s}, 3 \mathrm{H}), 2.62(\mathrm{~s}, 3 \mathrm{H}), 7.17(\mathrm{dd}, J=8.0,0.8$ $\mathrm{Hz}, 1 \mathrm{H}), 7.43(\mathrm{~d}, J=8.0 \mathrm{~Hz}, 1 \mathrm{H}), 7.45(\mathrm{~d}, J=0.8 \mathrm{~Hz}, 1 \mathrm{H}) ;{ }^{13} \mathrm{C}$ NMR $\left(101 \mathrm{MHz}, \mathrm{CDCl}_{3}\right) \delta 21.0\left(\mathrm{CH}_{3}\right), 30.2\left(\mathrm{CH}_{3}\right), 119.3(\mathrm{C}), 128.2$ (CH), $129.4(\mathrm{CH}), 134.5(\mathrm{CH}), 138.1(\mathrm{C}), 142.9(\mathrm{C}), 200.7$ (C); MS (CI) $m / z 215\left(\mathrm{MH}^{+}, 48\right), 213$ (51), 136 (100), 71 (33), 69 (35).

1-(2'-Bromo-4',5'-methylenedioxyphenyl)ethan-1-one (3c). ${ }^{28}$ The reaction was performed according to general procedure $B$ using 1-(2'-bromo-4',5'-methylenedioxyphenyl)ethan-1-ol (13c) (0.50 g, $2.0 \mathrm{mmol})$. Purification by flash column chromatography eluting with $10 \%$ diethyl ether in petroleum ether $(40-60)$ gave $1-\left(2^{\prime}\right.$-bromo$4^{\prime}, 5^{\prime}$-methylenedioxyphenyl)ethan-1-one (3c) (0.39 g, 79\%) as a colorless oil. Spectroscopic data were consistent with the literature: ${ }^{28}$ ${ }^{1} \mathrm{H}$ NMR $\left(400 \mathrm{MHz}, \mathrm{CDCl}_{3}\right) \delta 2.61(\mathrm{~s}, 3 \mathrm{H}), 6.04(\mathrm{~s}, 2 \mathrm{H}), 7.03(\mathrm{~s}$, $1 \mathrm{H}), 7.05(\mathrm{~s}, 1 \mathrm{H}) ;{ }^{13} \mathrm{C} \mathrm{NMR}\left(101 \mathrm{MHz}, \mathrm{CDCl}_{3}\right) \delta 30.3\left(\mathrm{CH}_{3}\right), 102.5$ $\left(\mathrm{CH}_{2}\right), 109.3(\mathrm{CH}), 112.0(\mathrm{C}), 113.9(\mathrm{CH}), 134.3(\mathrm{C}), 147.4(\mathrm{C})$, 150.4 (C), 199.5 (C); MS (ESI) $m / z 267\left(\mathrm{MNa}^{+}, 100\right), 265$ (99).

1-(2'-Bromo-5'-methoxyphenyl)ethan-1-one (3d). ${ }^{10}$ The reaction was performed according to general procedure A using 1-( $2^{\prime}$ bromo-5'-methoxyphenyl)ethan-1-ol (13d) (0.62 g, $2.7 \mathrm{mmol})$. Purification by flash column chromatography eluting with $10 \%$ diethyl ether in petroleum ether $(40-60)$ gave $1-\left(2^{\prime}\right.$-bromo- $5^{\prime}$ methoxyphenyl)ethan-1-one (3d) (0.46 g, 74\%) as a colorless oil. Spectroscopic data were consistent with the literature: ${ }^{10}{ }^{1} \mathrm{H}$ NMR $\left(400 \mathrm{MHz}, \mathrm{CDCl}_{3}\right) \delta 2.63(\mathrm{~s}, 3 \mathrm{H}), 3.81(\mathrm{~s}, 3 \mathrm{H}), 6.85(\mathrm{dd}, J=8.8,3.1$ $\mathrm{Hz}, 1 \mathrm{H}), 6.98(\mathrm{~d}, J=3.1 \mathrm{~Hz}, 1 \mathrm{H}), 7.48(\mathrm{~d}, J=8.8 \mathrm{~Hz}, 1 \mathrm{H}) ;{ }^{13} \mathrm{C}$ NMR $\left(101 \mathrm{MHz}, \mathrm{CDCl}_{3}\right) \delta 30.3\left(\mathrm{CH}_{3}\right), 55.6\left(\mathrm{CH}_{3}\right), 109.1(\mathrm{C}), 114.2$ (CH), $117.9(\mathrm{CH}), 134.6(\mathrm{CH}), 142.3(\mathrm{C}), 158.9$ (C), $201.3(\mathrm{C})$; MS (EI) $\mathrm{m} / z 230\left(\mathrm{M}^{+}, 54\right), 228(55), 215$ (99), 213 (100), 187 (17), 185 (17), 172 (14), 157 (15), 78 (18), 63 (35).

1-(2'-Bromo-5'-fluorophenyl)ethan-1-one (3e). ${ }^{10}$ The reaction was performed according to general procedure A using 1-( $2^{\prime}$ bromo-5'-fluorophenyl)ethan-1-ol (13e) (0.55 g, $2.5 \mathrm{mmol})$. Purification by flash column chromatography eluting with $10 \%$ diethyl ether in petroleum ether (40-60) gave 1-( $2^{\prime}$-bromo-5'-fluorophenyl)ethan-1-one (3e) $(0.40 \mathrm{~g}, 74 \%)$ as a colorless oil. Spectroscopic data were consistent with the literature: ${ }^{10}{ }^{1} \mathrm{H}$ NMR $\left(400 \mathrm{MHz}, \mathrm{CDCl}_{3}\right) \delta$ $2.64(\mathrm{~s}, 3 \mathrm{H}), 7.04$ (ddd, $J=8.8,7.6,3.1 \mathrm{~Hz}, 1 \mathrm{H}), 7.18$ (dd, $J=8.4,3.1$ $\mathrm{Hz}, 1 \mathrm{H}), 7.58(\mathrm{dd}, J=8.8,4.9 \mathrm{~Hz}, 1 \mathrm{H}) ;{ }^{13} \mathrm{C} \mathrm{NMR}\left(101 \mathrm{MHz}, \mathrm{CDCl}_{3}\right)$ $\delta 30.1\left(\mathrm{CH}_{3}\right), 113.1\left(\mathrm{~d},{ }^{4} J_{\mathrm{C}-\mathrm{F}}=3.2 \mathrm{~Hz}, \mathrm{C}\right), 116.1\left(\mathrm{~d},{ }^{2} J_{\mathrm{C}-\mathrm{F}}=24.1 \mathrm{~Hz}\right.$, 
$\mathrm{CH}), 119.1\left(\mathrm{~d},{ }^{2} J_{\mathrm{C}-\mathrm{F}}=22.5 \mathrm{~Hz}, \mathrm{CH}\right), 135.4\left(\mathrm{~d},{ }^{3} J_{\mathrm{C}-\mathrm{F}}=7.8 \mathrm{~Hz}, \mathrm{CH}\right)$, $142.9\left(\mathrm{~d},{ }^{3} \mathrm{~J}_{\mathrm{C}-\mathrm{F}}=5.8 \mathrm{~Hz}, \mathrm{C}\right), 161.4\left(\mathrm{~d},{ }^{1} J_{\mathrm{C}-\mathrm{F}}=250.3 \mathrm{~Hz}, \mathrm{C}\right), 199.9$ (C); MS (EI) $m / z 218\left(\mathrm{M}^{+}, 35\right), 216$ (36), 203 (98), 201 (100), 175 (33), 173 (34), 94 (58), 86 (38), 84 (60).

1 -(2'-Bromo-5'-chlorophenyl)ethan-1-one (3f). ${ }^{24}$ The reaction was performed according to general procedure A using 1-(2'-bromo5'-chlorophenyl)ethan-1-ol (13f) $(0.43 \mathrm{~g}, 1.8 \mathrm{mmol})$. Purification by flash column chromatography eluting with $10 \%$ diethyl ether in petroleum ether (40-60) gave 1-(2'-bromo-5'-chlorophenyl)ethan-1one (3f) $(0.33 \mathrm{~g}, 78 \%)$ as a yellow oil. Spectroscopic data were consistent with the literature: ${ }^{24}{ }^{1} \mathrm{H}$ NMR $\left(400 \mathrm{MHz}, \mathrm{CDCl}_{3}\right) \delta 2.63$ $(\mathrm{s}, 3 \mathrm{H}), 7.28(\mathrm{dd}, J=8.5,2.5 \mathrm{~Hz}, 1 \mathrm{H}), 7.43(\mathrm{~d}, J=2.5 \mathrm{~Hz}, 1 \mathrm{H}), 7.55$ $(\mathrm{d}, J=8.5 \mathrm{~Hz}, 1 \mathrm{H}) ;{ }^{13} \mathrm{C}$ NMR $\left(101 \mathrm{MHz}, \mathrm{CDCl}_{3}\right) \delta 30.2\left(\mathrm{CH}_{3}\right)$, $116.8(\mathrm{C}), 128.9(\mathrm{CH}), 131.8(\mathrm{CH}), 133.8(\mathrm{C}), 135.0(\mathrm{CH}), 142.7$ (C), 199.9 (C); MS (EI) m/z $234\left(\mathrm{M}^{+}, 45\right), 232$ (33), 219 (100), 217 (77), 191 (39), 189 (24), 110 (16), 84 (12), 75 (33).

1-(2'-Bromo-3'-pyridyl)ethan-1-one (3h). To a stirred solution of 1-(2'-bromo-3'-pyridyl)ethan-1-ol (13h) (0.940 g, $4.70 \mathrm{mmol})$ in DMF $(5 \mathrm{~mL})$ at room temperature was added a solution of PDC $(2.65$ $\mathrm{g}, 7.05 \mathrm{mmol})$ in DMF $(5 \mathrm{~mL})$. The resulting suspension was stirred for $18 \mathrm{~h}$, filtered through a pad of Celite, washed with diethyl ether (2 $\times 30 \mathrm{~mL}$ ), and concentrated in vacuo. The crude product was then purified using silica gel flash column chromatography eluting with $10 \%$ diethyl ether in petroleum ether $(40-60)$ to give 1 - $\left(2^{\prime}\right.$-bromo- $3^{\prime}$ pyridyl)ethan-1-one (3h) (0.69 g, 74\%) as a yellow oil: IR (neat) 1697, 1566, 1389, $1273 \mathrm{~cm}^{-1}$; ${ }^{1} \mathrm{H}$ NMR $\left(400 \mathrm{MHz}, \mathrm{CDCl}_{3}\right) \delta 2.68(\mathrm{~s}$, $3 \mathrm{H}), 7.36(\mathrm{dd}, J=7.6,4.7 \mathrm{~Hz}, 1 \mathrm{H}), 7.75(\mathrm{dd}, J=7.6,2.0 \mathrm{~Hz}, 1 \mathrm{H}), 8.45$ $(\mathrm{dd}, J=4.7,2.0 \mathrm{~Hz}, 1 \mathrm{H}) ;{ }^{13} \mathrm{C} \mathrm{NMR}\left(101 \mathrm{MHz}, \mathrm{CDCl}_{3}\right) \delta 30.3\left(\mathrm{CH}_{3}\right)$, $122.7(\mathrm{CH}), 137.4(\mathrm{CH}), 138.1(\mathrm{C}), 138.5(\mathrm{C}), 151.4(\mathrm{CH}), 199.8$ (C); MS (ESI) $\mathrm{m} / z 200\left(\mathrm{MH}^{+}, 100\right)$; HRMS (ESI) calcd for $\mathrm{C}_{7} \mathrm{H}_{7}{ }^{79} \mathrm{BrNO}\left(\mathrm{MH}^{+}\right)$199.9706, found 199.9710.

$2^{\prime}$-Bromopropiophenone (3i). ${ }^{25}$ The reaction was performed according to general procedure B using 1-(2'-bromophenyl)propan-1ol (13i) (1.30 g, $6.00 \mathrm{mmol})$. Purification by flash column chromatography eluting with $10 \%$ diethyl ether in petroleum ether (40-60) gave $2^{\prime}$-bromopropiophenone (3i) (1.15 g, 90\%) as a yellow oil. Spectroscopic data were consistent with the literature: ${ }^{25} \delta_{\mathrm{H}}(400$ $\left.\mathrm{MHz}, \mathrm{CDCl}_{3}\right) \delta 1.21(\mathrm{t}, J=7.2 \mathrm{~Hz}, 3 \mathrm{H}), 2.93(\mathrm{q}, J=7.2 \mathrm{~Hz}, 2 \mathrm{H})$, $7.25-7.31(\mathrm{~m}, 1 \mathrm{H}), 7.33-7.39(\mathrm{~m}, 2 \mathrm{H}), 7.57-7.62(\mathrm{~m}, 1 \mathrm{H}) ;{ }^{13} \mathrm{C}$ NMR (101 MHz, CDCl $) \delta 8.1\left(\mathrm{CH}_{3}\right), 36.1\left(\mathrm{CH}_{2}\right), 118.5(\mathrm{C}), 127.4$ $(\mathrm{CH}), 128.2(\mathrm{CH}), 131.3(\mathrm{CH}), 133.6(\mathrm{CH}), 142.0(\mathrm{C}), 205.1(\mathrm{C})$; MS (ESI) $m / z 237\left(\mathrm{MNa}^{+}, 98\right) 235$ (100).

1-(2'-Bromophenyl)-2-methylpropan-1-one $(3 j) .^{26}$ The reaction was performed according to general procedure $B$ using 1- $\left(2^{\prime}-\right.$ bromophenyl)-2-methylpropan-1-ol (13j) (0.90 g, $3.9 \mathrm{mmol})$. Purification by flash column chromatography eluting with $10 \%$ diethyl ether in petroleum ether $(40-60)$ gave 1-( $2^{\prime}$-bromophenyl)-2methylpropan-1-one (3j) $(0.73 \mathrm{~g}, 83 \%)$ as a yellow oil. Spectroscopic data were consistent with the literature: ${ }^{26}{ }^{1} \mathrm{H}$ NMR $(400 \mathrm{MHz}$, $\left.\mathrm{CDCl}_{3}\right) \delta 1.20(\mathrm{~d}, J=7.0 \mathrm{~Hz}, 6 \mathrm{H}), 3.32$ (sept, $\left.J=7.0 \mathrm{~Hz}, 1 \mathrm{H}\right), 7.25-$ $7.31(\mathrm{~m}, 2 \mathrm{H}), 7.33-7.39(\mathrm{~m}, 1 \mathrm{H}), 7.57-7.62(\mathrm{~m}, 1 \mathrm{H}) ;{ }^{13} \mathrm{C}$ NMR $\left(101 \mathrm{MHz}, \mathrm{CDCl}_{3}\right) \delta 18.1\left(2 \times \mathrm{CH}_{3}\right), 40.2(\mathrm{CH}), 118.7(\mathrm{C}), 127.2$ $(\mathrm{CH}), 128.1(\mathrm{CH}), 131.0(\mathrm{CH}), 133.4(\mathrm{CH}), 142.1(\mathrm{C}), 208.7(\mathrm{C})$; MS (ESI) $m / z 251\left(\mathrm{MNa}^{+}, 98\right) 249$ (100).

1-(2'-Bromo-5'-nitrophenyl)ethan-1-one $(3 \mathrm{~g}) .^{22} 1-\left(2^{\prime}-\right.$ Bromophenyl)ethan-1-one (3a) $(0.54 \mathrm{~mL}, 4.0 \mathrm{mmol})$ was added to a solution of potassium nitrate $(0.50 \mathrm{~g}, 5.0 \mathrm{mmol})$ in concentrated sulfuric acid $(4 \mathrm{~mL})$ at $0{ }^{\circ} \mathrm{C}$. The reaction mixture was then allowed to warm to room temperature and stirred for $1.5 \mathrm{~h}$. The reaction was quenched with water $(5 \mathrm{~mL})$ and the mixture extracted with dichloromethane $(2 \times 15 \mathrm{~mL})$, dried over $\mathrm{MgSO}_{4}$, filtered, and concentrated in vacuo. Purification by silica gel flash chromatography eluting with $10 \%$ diethyl ether in petroleum ether $(40-60)$ yielded 1(2'-bromo-5'-nitrophenyl)ethan-1-one $(3 \mathrm{~g})(0.53 \mathrm{~g}, 54 \%)$ as a white powder: $\mathrm{mp} 86-87{ }^{\circ} \mathrm{C}$ (lit. $\left.{ }^{22} 85-87{ }^{\circ} \mathrm{C}\right) ;{ }^{1} \mathrm{H}$ NMR $(400 \mathrm{MHz}$, $\left.\mathrm{CDCl}_{3}\right) \delta 2.69(\mathrm{~s}, 3 \mathrm{H}), 7.83(\mathrm{~d}, J=8.7 \mathrm{~Hz}, 1 \mathrm{H}), 8.15(\mathrm{dd}, J=8.7,2.7$ $\mathrm{Hz}, 1 \mathrm{H}), 8.31(\mathrm{~d}, J=2.7 \mathrm{~Hz}, 1 \mathrm{H}) ;{ }^{13} \mathrm{C} \mathrm{NMR}\left(101 \mathrm{MHz}, \mathrm{CDCl}_{3}\right) \delta$ $30.1\left(\mathrm{CH}_{3}\right), 123.8(\mathrm{CH}), 125.9(\mathrm{CH}), 126.2(\mathrm{C}), 135.2(\mathrm{CH}), 142.4$ (C), 147.1 (C), 198.7 (C); MS (EI) $m / z 245\left(\mathrm{M}^{+}, 27\right), 243$ (27), 230 (100), 182 (22), 151 (28), 105 (17), 75 (44).
B-Allyl-1,3,2-dioxaborinane (6). ${ }^{15 \mathrm{c}}$ Trimethylborate $(5.6 \mathrm{~mL}, 50$ $\mathrm{mmol})$ was dissolved in dry diethyl ether $(50 \mathrm{~mL})$ under an argon atmosphere and cooled to $-78{ }^{\circ} \mathrm{C}$. An allylmagnesium bromide solution ( $50 \mathrm{~mL}, 1 \mathrm{M}$ in diethyl ether) was added dropwise over $0.5 \mathrm{~h}$. After addition, the reaction mixture was stirred for $2 \mathrm{~h}$ at $-78^{\circ} \mathrm{C}$ and then acidified at $0{ }^{\circ} \mathrm{C}$ with a $3 \mathrm{M}$ aqueous solution of hydrochloric acid $(60 \mathrm{~mL})$. The organic phase was separated, and the aqueous layer was extracted with diethyl ether $(3 \times 50 \mathrm{~mL})$. The combined organic layers were dried over $\mathrm{MgSO}_{4}$, filtered, and concentrated in vacuo to approximately $100 \mathrm{~mL}$. To this solution was added 1,3-propanediol (3.6 mL, $50 \mathrm{mmol})$ as well as oven-dried 4 Å molecular sieves $(10 \mathrm{~g})$, and the resulting mixture was stirred for $16 \mathrm{~h}$ at room temperature. The molecular sieves were filtered and washed with diethyl ether $(2 \times$ $50 \mathrm{~mL}$ ). The solvent was removed in vacuo, and the crude product was then dissolved in $n$-pentane $(100 \mathrm{~mL})$. The resulting cloudy suspension was filtered through a pad of Celite, washed, and concentrated in vacuo. Purification by silica gel flash chromatography eluting with $35 \%$ diethyl ether in $n$-pentane gave a pale yellow oil. Further purification by short-path Kugelrohr distillation gave $B$-allyl1,3,2-dioxaborinane (6) (4.0 g, 63\%) as a colorless oil. Spectroscopic data were consistent with the literature: ${ }^{15 c}{ }^{1} \mathrm{H}$ NMR $(400 \mathrm{MHz}$, $\mathrm{CDCl}_{3}$ ) $\delta 1.63$ (br d, $J=7.6 \mathrm{~Hz}, 2 \mathrm{H}$ ), 1.93 (quin, $J=5.5 \mathrm{~Hz}, 2 \mathrm{H}$ ), 3.98 $(\mathrm{t}, J=5.5 \mathrm{~Hz}, 4 \mathrm{H}), 4.88(\mathrm{ddt}, J=10.0,2.4,1.2 \mathrm{~Hz}, 1 \mathrm{H}), 4.92(\mathrm{ddt}, J=$ $15.2,2.4,1.2 \mathrm{~Hz}, 1 \mathrm{H}), 5.85(\mathrm{ddt}, J=15.2,10.0,7.6 \mathrm{~Hz}, 1 \mathrm{H}) ;{ }^{13} \mathrm{C}$ NMR $\left(101 \mathrm{MHz}, \mathrm{CDCl}_{3}\right) \delta 22.0\left(\mathrm{~d},{ }^{1} \mathrm{~J}_{\mathrm{C}-\mathrm{B}}=64.6 \mathrm{~Hz}, \mathrm{CH}_{2}\right), 27.3\left(\mathrm{CH}_{2}\right)$, $61.9\left(2 \times \mathrm{CH}_{2}\right), 113.9\left(\mathrm{CH}_{2}\right) 135.5(\mathrm{CH}) ; \mathrm{MS}(\mathrm{CI}) \mathrm{m} / z 127\left(\mathrm{MH}^{+}\right.$, 12), 113 (32), 103 (44), 97 (40), 85 (83), 71 (100), 69 (71).

(2S)-2-(2'-Bromophenyl)pent-4-en-2-ol (4). ${ }^{10}$ B-Allyl-1,3,2-dioxaborinane $(6)(0.026 \mathrm{~mL}, 0.21 \mathrm{mmol})$ and $(S)-(-)-3,3^{\prime}$-dibromo1,1'-bi-2-naphthol (9) $(0.0050 \mathrm{~g}, 0.011 \mathrm{mmol})$ was dissolved in toluene $(1 \mathrm{~mL})$ and stirred for $0.1 \mathrm{~h}$ at $35{ }^{\circ} \mathrm{C}$ in an oven-dried microwave vial. A solution of 1-(2'-bromophenyl)ethan-1-one (3a) $(0.020 \mathrm{~mL}, 0.15 \mathrm{mmol})$ in distilled toluene $(0.75 \mathrm{~mL})$ was added, and the reaction mixture was stirred for 5 days at $35^{\circ} \mathrm{C}$ under an argon atmosphere. Purification by flash column chromatography eluting with $10 \%$ diethyl ether in petroleum ether $(40-60)$ gave $(2 S)-2-(2$ bromophenyl)pent-4-en-2-ol (4) (0.036 g, 100\%) as a colorless oil. Spectroscopic data were consistent with the literature: ${ }^{10}[\alpha]_{\mathrm{D}}{ }^{31}-34.3$ (c 1.2, $\mathrm{CHCl}_{3}$ ); ${ }^{1} \mathrm{H}$ NMR (400 MHz, $\left.\mathrm{CDCl}_{3}\right) \delta 1.72(\mathrm{~s}, 3 \mathrm{H}), 2.62$ (s, $1 \mathrm{H}), 2.64$ (br dd, $J=14.0,8.4 \mathrm{~Hz}, 1 \mathrm{H}), 3.29$ (ddt $J=14.0,6.3,1.1 \mathrm{~Hz}$, $1 \mathrm{H}), 5.06-5.19(\mathrm{~m}, 2 \mathrm{H}), 5.55$ (dddd, $J=17.0,10.1,8.4,6.3 \mathrm{~Hz}, 1 \mathrm{H})$, $7.10(\mathrm{td}, J=7.8,1.7 \mathrm{~Hz}, 1 \mathrm{H}), 7.30(\mathrm{td}, J=7.8,1.3 \mathrm{~Hz}, 1 \mathrm{H}), 7.58(\mathrm{dd}, J$ $=7.8,1.3 \mathrm{~Hz}, 1 \mathrm{H}), 7.70(\mathrm{dd}, J=7.8,1.7 \mathrm{~Hz}, 1 \mathrm{H}) ;{ }^{13} \mathrm{C} \mathrm{NMR}(101$ $\left.\mathrm{MHz}, \mathrm{CDCl}_{3}\right) \delta 27.3\left(\mathrm{CH}_{3}\right), 45.1\left(\mathrm{CH}_{2}\right), 74.7(\mathrm{C}), 119.4\left(\mathrm{CH}_{2}\right)$, $120.0(\mathrm{C}), 127.4(\mathrm{CH}), 128.3(\mathrm{CH}), 128.6(\mathrm{CH}), 133.7(\mathrm{CH}), 135.1$ (CH), 145.0 (C); MS (ESI) $m / z 265\left(\mathrm{MNa}^{+}, 100\right), 263$ (97). The enantiomeric excess was determined by HPLC analysis with a chiralcel OD-H column (95:5 hexane $/{ }^{i} \mathrm{PrOH}$, flow rate of $\left.1.0 \mathrm{~mL} / \mathrm{min}\right): t_{\text {minor }}=$ $1.78 \mathrm{~min}, t_{\text {major }}=1.96 \mathrm{~min} ; 98: 2 \mathrm{er}$.

General Procedure for the One-Pot Asymmetric Synthesis of 1-Alkyl-1-indanols. B-Allyl-1,3,2-dioxaborinane (6) (1.4 equiv) and $(S)$-(-)-3,3'-dibromo-1,1'-bi-2-naphthol (9) (7.5 mol \%) were dissolved in distilled toluene $(5 \mathrm{~mL} / \mathrm{mmol})$ and stirred for $0.1 \mathrm{~h}$ at 35 ${ }^{\circ} \mathrm{C}$ in an oven-dried microwave vial. A solution of 2 -bromoaryl ketone (1.0 equiv) in toluene $(5 \mathrm{~mL} / \mathrm{mmol})$ was added, and the reaction mixture was stirred for 5 days at $35{ }^{\circ} \mathrm{C}$. Bis(triphenylphosphine)palladium(II) dichloride $(7.5 \mathrm{~mol} \%)$, potassium carbonate (2 equiv), and hydrazine monohydrate ( 0.4 equiv) were added to the reaction mixture. The vial was sealed and heated to $135{ }^{\circ} \mathrm{C}$ for $18 \mathrm{~h}$. The reaction mixture was cooled to room temperature, diluted with diethyl ether $(2 \mathrm{~mL} / \mathrm{mmol})$, filtered through a pad of Celite, and concentrated in vacuo. Purification of the crude product using silica gel flash chromatography eluting with diethyl ether or ethyl acetate in petroleum ether or ethyl acetate in hexane gave the corresponding 1-alkyl-1-indenols.

(1S)-1,3-Dimethylinden-1-ol (S-11a) and (1S)-2,3-Dihydro-1methyl-3-(methylene)indan-1-ol $(S-10 a) .{ }^{10}$ The reaction was performed according to the general procedure using $1-\left(2^{\prime}\right.$ bromophenyl)ethan-1-one (3a) $(0.067 \mathrm{~mL}, 0.50 \mathrm{mmol})$. The crude mixture was purified by silica gel flash chromatography eluting with 
$10 \%$ diethyl ether in petroleum ether $(40-60)$ to yield first $(1 S)-1,3-$ dimethylinden-1-ol (S-11a) (0.012 g, 15\%) and then (1S)-2,3-dihydro1-methyl-3-(methylene)indan-1-ol ( $\boldsymbol{S}$-10a) $(0.060 \mathrm{~g}, 75 \%)$ as colorless oils, which solidified upon standing. Spectroscopic data were consistent with the literature. ${ }^{10}$ Data for $(1 S)$-1,3-dimethylinden-1-ol (S-11a): mp 95-97 ${ }^{\circ} \mathrm{C} ;[\alpha]_{\mathrm{D}}{ }^{31}+55.9\left(\right.$ c 1.0, $\left.\mathrm{CHCl}_{3}\right)$; ${ }^{1} \mathrm{H}$ NMR $(400$ $\left.\mathrm{MHz}, \mathrm{CDCl}_{3}\right) \delta 1.58(\mathrm{~s}, 3 \mathrm{H}), 1.67(\mathrm{~s}, 1 \mathrm{H}), 2.06(\mathrm{~d}, J=1.6 \mathrm{~Hz}, 3 \mathrm{H})$, $5.99(\mathrm{q}, J=1.6 \mathrm{~Hz}, 1 \mathrm{H}), 7.15(\mathrm{br} \mathrm{d}, J=7.3 \mathrm{~Hz}, 1 \mathrm{H}), 7.22(\mathrm{td}, J=7.3$, $1.0 \mathrm{~Hz}, 1 \mathrm{H}), 7.28(\mathrm{td}, J=7.3,1.0 \mathrm{~Hz}, 1 \mathrm{H}), 7.40(\mathrm{br} \mathrm{d}, J=7.3 \mathrm{~Hz}, 1 \mathrm{H})$; ${ }^{13} \mathrm{C}$ NMR (101 MHz, CDCl $) \delta 12.7\left(\mathrm{CH}_{3}\right), 23.8\left(\mathrm{CH}_{3}\right), 81.1(\mathrm{C})$, $119.3(\mathrm{CH}), 121.2(\mathrm{CH}), 126.3(\mathrm{CH}), 128.3(\mathrm{CH}), 137.7(\mathrm{CH})$, 138.9 (C), 143.0 (C), 150.0 (C); MS (ESI) $m / z 183\left(\mathrm{MNa}^{+}, 100\right)$. The enantiomeric excess was determined by HPLC analysis with a chiralcel OD-H column (95:5 hexane $/{ }^{i} \mathrm{PrOH}$, flow rate of $1.0 \mathrm{~mL} /$ $\min ): t_{\text {major }}=2.43 \mathrm{~min}, t_{\text {minor }}=2.80 \mathrm{~min}$; 96:4 er. Data for $(1 S)-2,3-$ dihydro-1-methyl-3-(methylene)indan-1-ol (S-10a): mp 79-81 ${ }^{\circ} \mathrm{C}$; $[\alpha]_{\mathrm{D}}{ }^{31}+10.9\left(\mathrm{c} \mathrm{1.0}, \mathrm{CHCl}_{3}\right) ;{ }^{1} \mathrm{H} \mathrm{NMR}\left(400 \mathrm{MHz}, \mathrm{CDCl}_{3}\right) \delta 1.59(\mathrm{~s}$, $3 \mathrm{H}), 1.95(\mathrm{~s}, 1 \mathrm{H}), 2.88(\mathrm{dt}, J=16.5,2.1 \mathrm{~Hz}, 1 \mathrm{H}), 2.95(\mathrm{dt}, J=16.5$, $2.1 \mathrm{~Hz}, 1 \mathrm{H}), 5.09(\mathrm{t}, J=2.1 \mathrm{~Hz}, 1 \mathrm{H}), 5.52(\mathrm{t}, J=2.1 \mathrm{~Hz}, 1 \mathrm{H}), 7.28-$ $7.34(\mathrm{~m}, 2 \mathrm{H}), 7.39-7.44(\mathrm{~m}, 1 \mathrm{H}), 7.46-7.52(\mathrm{~m}, 1 \mathrm{H}) ;{ }^{13} \mathrm{C}$ NMR $\left(101 \mathrm{MHz}, \mathrm{CDCl}_{3}\right) \delta 28.1\left(\mathrm{CH}_{3}\right), 49.4\left(\mathrm{CH}_{2}\right), 78.7(\mathrm{C}), 104.5\left(\mathrm{CH}_{2}\right)$, $120.6(\mathrm{CH}), 122.9(\mathrm{CH}), 128.6(\mathrm{CH}), 129.1(\mathrm{CH}), 139.4(\mathrm{C}), 145.9$ (C), 150.4 (C); MS (ESI) $m / z 183\left(\mathrm{MNa}^{+}, 100\right)$. The enantiomeric excess was determined by HPLC analysis with a chiralcel OD-H column $\left(95: 5\right.$ hexane $/{ }^{i} \mathrm{PrOH}$, flow rate of $\left.1.0 \mathrm{~mL} / \mathrm{min}\right): t_{\text {major }}=2.58$ $\min , t_{\text {minor }}=2.96 \mathrm{~min} ; 96: 4 \mathrm{er}$.

(1R)-1,3-Dimethylinden-1-ol (R-11a) and (1R)-2,3-Dihydro-1methyl-3-(methylene)indan-1-ol $(R-10 a)$. The reaction was performed according to the general procedure using 1-( $2^{\prime}$ bromophenyl)ethan-1-one (3a) $(0.067 \mathrm{~mL}, 0.50 \mathrm{mmol})$ and $(R)$ (+)-3,3'-dibromo-1,1'-bi-2-naphthol (0.016 g, $0.040 \mathrm{mmol})$. The crude mixture was purified by silica gel flash chromatography eluting with $10 \%$ diethyl ether in petroleum ether $(40-60)$ to yield first $(1 R)$ 1,3-dimethylinden-1-ol (R-11a) (0.014 g, 18\%) and then (1R)-2,3dihydro-1-methyl-3-(methylene)indan-1-ol (R-10a) $(0.056 \mathrm{~g}, 70 \%)$ as colorless oils, which solidified upon standing. Melting point and spectroscopic data were as recorded for $S$-11a and $S$-10a. Additional data for R-11a: $[\alpha]_{\mathrm{D}}{ }^{31}-56.2\left(c 0.5, \mathrm{CHCl}_{3}\right)$. The enantiomeric excess was determined by HPLC analysis with a chiralcel OD-H column (95:5 hexane $/{ }^{i} \mathrm{PrOH}$, flow rate of $1.0 \mathrm{~mL} / \mathrm{min}$ ): $t_{\text {minor }}=2.38 \mathrm{~min}, t_{\text {major }}$ $=2.75 \mathrm{~min} ; 96: 4$ er. Additional data for R-10a: $[\alpha]_{\mathrm{D}}{ }^{31}-13.0(c$ 1.0, $\mathrm{CHCl}_{3}$ ). The enantiomeric excess was determined by HPLC analysis with a chiralcel OD-H column (95:5 hexane $/{ }^{i} \mathrm{PrOH}$, flow rate of 1.0 $\mathrm{mL} / \mathrm{min}): t_{\text {minor }}=2.53 \mathrm{~min}, t_{\text {major }}=2.94 \mathrm{~min} ; 96: 4$ er.

(1S)-2,3-Dihydro-1,5-dimethyl-3-(methylene)indan-1-ol (10b). The reaction was performed according to the general procedure using 1-(2'-bromo-4'-methylphenyl)ethan-1-one (3b) ( $0.10 \mathrm{~g}, 0.47$ $\mathrm{mmol})$. The crude mixture was purified by silica gel flash chromatography eluting with $8 \%$ ethyl acetate in hexane to yield (1S)-2,3-dihydro-1,5-dimethyl-3-(methylene)indan-1-ol (10b) (0.050 $\mathrm{g}, 61 \%)$ as a colorless oil: IR (neat) $3352,2969,1644 \mathrm{~cm}^{-1} ;[\alpha]_{\mathrm{D}}{ }^{31}$ +24.4 (c 1.2, $\left.\mathrm{CHCl}_{3}\right) ;{ }^{1} \mathrm{H}$ NMR (400 MHz, $\left.\mathrm{CDCl}_{3}\right) \delta 1.56(\mathrm{~s}, 3 \mathrm{H})$, $1.90(\mathrm{~s}, 1 \mathrm{H}), 2.37(\mathrm{~s}, 3 \mathrm{H}), 2.87(\mathrm{dt}, J=16.4,2.1 \mathrm{~Hz}, 1 \mathrm{H}), 2.93(\mathrm{dt}, J=$ 16.4, $2.1 \mathrm{~Hz}, 1 \mathrm{H}), 5.05(\mathrm{t}, J=2.1 \mathrm{~Hz}, 1 \mathrm{H}), 5.48(\mathrm{t}, J=2.1 \mathrm{~Hz}, 1 \mathrm{H})$, $7.13($ br d, $J=7.9 \mathrm{~Hz}, 1 \mathrm{H}), 7.28-7.32(\mathrm{~m}, 2 \mathrm{H}) ;{ }^{13} \mathrm{C} \mathrm{NMR}(101 \mathrm{MHz}$, $\left.\mathrm{CDCl}_{3}\right) \delta 21.4\left(\mathrm{CH}_{3}\right), 28.1\left(\mathrm{CH}_{3}\right), 49.6\left(\mathrm{CH}_{2}\right), 78.4(\mathrm{C}), 104.1$ $\left(\mathrm{CH}_{2}\right), 121.0(\mathrm{CH}), 122.7(\mathrm{CH}), 130.1(\mathrm{CH}), 138.5(\mathrm{C}), 139.6(\mathrm{C})$, 146.0 (C), 147.8 (C); MS (ESI) $m / z 197\left(\mathrm{MNa}^{+}, 100\right)$; HRMS (ESI) calcd for $\mathrm{C}_{12} \mathrm{H}_{14} \mathrm{NaO}\left(\mathrm{MNa}^{+}\right)$197.0937, found 197.0933. The enantiomeric excess was determined by HPLC analysis with a chiralcel OD-H column (99:1 hexane $/{ }^{i} \mathrm{PrOH}$, flow rate of $\left.1.0 \mathrm{~mL} / \mathrm{min}\right): t_{\text {minor }}=$ $12.88 \mathrm{~min}, t_{\mathrm{major}}=13.57 \mathrm{~min} ; 96: 4 \mathrm{er}$.

(1S)-2,3-Dihydro-5,6-(methylenedioxy)-1-methyl-3(methylene)indan-1-ol (10c). The reaction was performed according to the general procedure using 1-( $2^{\prime}$-bromo- $4^{\prime}, 5^{\prime}$ methylenedioxyphenyl)ethan-1-one (3c) (0.12 g, $0.50 \mathrm{mmol})$. The crude mixture was purified by silica gel flash chromatography eluting with $20 \%$ diethyl ether in petroleum ether $(40-60)$ to yield $(1 S)-2,3-$ dihydro-5,6-(methylenedioxy)-1-methyl-3-(methylene)indan-1-ol (10c) $(0.071 \mathrm{~g}, 69 \%)$ as a colorless oil: IR (neat) 3403, 2970, 1474 $\mathrm{cm}^{-1} ;[\alpha]_{\mathrm{D}}{ }^{30}+35.3\left(c\right.$ 1.0, MeOH); ${ }^{1} \mathrm{H}$ NMR (400 MHz, $\left.\mathrm{CD}_{3} \mathrm{OD}\right) \delta$ $1.45(\mathrm{~s}, 3 \mathrm{H}), 2.86(\mathrm{t}, J=2.0 \mathrm{~Hz}, 2 \mathrm{H}), 4.89(\mathrm{t}, J=2.0 \mathrm{~Hz}, 1 \mathrm{H}), 5.29(\mathrm{t}$, $J=2.0 \mathrm{~Hz}, 1 \mathrm{H}), 5.94(\mathrm{~d}, J=1.2 \mathrm{~Hz}, 1 \mathrm{H}), 5.95(\mathrm{~d}, J=1.2 \mathrm{~Hz}, 1 \mathrm{H})$, $6.82(\mathrm{~s}, 1 \mathrm{H}), 6.90(\mathrm{~s}, 1 \mathrm{H}) ;{ }^{13} \mathrm{C}$ NMR $\left(101 \mathrm{MHz}, \mathrm{CD}_{3} \mathrm{OD}\right) \delta 27.6$ $\left(\mathrm{CH}_{3}\right), 49.0\left(\mathrm{CH}_{2}\right), 77.4(\mathrm{C}), 99.6(\mathrm{CH}), 100.5\left(\mathrm{CH}_{2}\right), 101.4\left(\mathrm{CH}_{2}\right)$, 102.5 (CH), 133.4 (C), 145.3 (C), 146.0 (C), 148.7 (C), 149.0 (C); MS (ESI) $m / z 227\left(\mathrm{MNa}^{+}, 100\right)$; HRMS (ESI) calcd for $\mathrm{C}_{12} \mathrm{H}_{12} \mathrm{NaO}_{3}$ $\left(\mathrm{MNa}^{+}\right)$227.0679, found 227.0672. The enantiomeric excess was determined by HPLC analysis with a chiralcel OD-H column (95:5 hexane $/{ }^{i} \mathrm{PrOH}$, flow rate of $\left.1.0 \mathrm{~mL} / \mathrm{min}\right): t_{\text {minor }}=6.04 \mathrm{~min}, t_{\text {major }}=$ $6.59 \mathrm{~min} ; 89.5: 10.5 \mathrm{er}$.

(1S)-2,3-Dihydro-6-methoxy-1-methyl-3-(methylene)indan1-ol (10d). ${ }^{10}$ The reaction was performed according to the general procedure using 1-(2'-bromo- $5^{\prime}$-methoxyphenyl)ethan-1-one (3d) $(0.12 \mathrm{~g}, 0.50 \mathrm{mmol})$. The crude mixture was purified by silica gel flash chromatography eluting with $20 \%$ diethyl ether in petroleum ether (40-60) to yield (1S)-2,3-dihydro-6-methoxy-1-methyl-3(methylene)indan-1-ol (10d) (0.044 g, 47\%) as a yellow oil. Spectroscopic data were consistent with the literature: ${ }^{10}[\alpha]_{\mathrm{D}}{ }^{30}$ +30.8 (c 1.0, MeOH); ${ }^{1} \mathrm{H}$ NMR (400 MHz, $\left.\mathrm{CD}_{3} \mathrm{OD}\right) \delta 1.48$ (s, $3 \mathrm{H}), 2.84(\mathrm{dt}, J=16.4,1.6 \mathrm{~Hz}, 1 \mathrm{H}), 2.89(\mathrm{dt}, J=16.4,1.6 \mathrm{~Hz}, 1 \mathrm{H})$, $3.80(\mathrm{~s}, 3 \mathrm{H}), 4.90(\mathrm{t}, J=1.6 \mathrm{~Hz}, 1 \mathrm{H}), 5.32(\mathrm{t}, J=1.6 \mathrm{~Hz}, 1 \mathrm{H}), 6.85$ $(\mathrm{dd}, J=8.5,2.3 \mathrm{~Hz}, 1 \mathrm{H}), 6.94(\mathrm{~d}, J=2.3 \mathrm{~Hz}, 1 \mathrm{H}), 7.39(\mathrm{~d}, J=8.5 \mathrm{~Hz}$, $1 \mathrm{H}) ;{ }^{13} \mathrm{C}$ NMR (101 MHz, CD $\left.\mathrm{OD}\right) \delta 27.7\left(\mathrm{CH}_{3}\right), 49.1\left(\mathrm{CH}_{2}\right), 54.5$ $\left(\mathrm{CH}_{3}\right), 77.6(\mathrm{C}), 100.4\left(\mathrm{CH}_{2}\right), 106.7(\mathrm{CH}), 115.2(\mathrm{CH}), 121.2(\mathrm{CH})$, 132.1 (C), 145.9 (C), 152.5 (C), 160.9 (C); MS (EI) m/z $190\left(\mathrm{M}^{+}\right.$, 84), 175 (100), 160 (10), 132 (11), 115 (14), 88 (12), 61 (17). The enantiomeric excess was determined by HPLC analysis with a chiralcel OD-H column (95:5 hexane $/{ }^{i} \mathrm{PrOH}$, flow rate of $\left.1.0 \mathrm{~mL} / \mathrm{min}\right): t_{\text {major }}=$ $2.99 \mathrm{~min}, t_{\text {minor }}=3.67 \mathrm{~min}$; 93.5:6.5 er.

(1S)-2,3-Dihydro-6-fluoro-1-methyl-3-(methylene)indan-1-ol (10e). The reaction was performed according to the general procedure using 1-(2'-bromo-5'-fluorophenyl)ethan-1-one (3e) (0.054 g, 0.25 $\mathrm{mmol})$. The crude mixture was purified by silica gel flash chromatography eluting with $10 \%$ diethyl ether in petroleum ether (40-60) to yield (1S)-2,3-dihydro-6-fluoro-1-methyl-3-(methylene)indan-1-ol (10e) $(0.020 \mathrm{~g}, 46 \%)$ as a colorless oil: IR (neat) 3356, 2970, 1643,1605, $1481 \mathrm{~cm}^{-1} ;[\alpha]_{\mathrm{D}}{ }^{31}+18.3\left(c 0.5, \mathrm{CHCl}_{3}\right)$; ${ }^{1} \mathrm{H}$ NMR $\left(400 \mathrm{MHz}, \mathrm{CD}_{3} \mathrm{OD}\right) \delta 1.48(\mathrm{~s}, 3 \mathrm{H}), 2.87(\mathrm{dt}, J=16.4,2.1 \mathrm{~Hz}, 1 \mathrm{H})$, $2.92(\mathrm{dt}, J=16.4,2.1 \mathrm{~Hz}, 1 \mathrm{H}), 5.02(\mathrm{t}, J=2.1 \mathrm{~Hz}, 1 \mathrm{H}), 5.45(\mathrm{t}, J=2.1$ $\mathrm{Hz}, 1 \mathrm{H}), 7.01$ (ddd, $J=9.2,8.6,2.4 \mathrm{~Hz}, 1 \mathrm{H}), 7.10(\mathrm{dd}, J=8.8,2.4 \mathrm{~Hz}$, $1 \mathrm{H}), 7.51(\mathrm{dd}, J=8.6,5.2 \mathrm{~Hz}, 1 \mathrm{H}) ;{ }^{13} \mathrm{C} \mathrm{NMR}\left(101 \mathrm{MHz}, \mathrm{CD}_{3} \mathrm{OD}\right) \delta$ $27.5\left(\mathrm{CH}_{3}\right), 48.9\left(\mathrm{CH}_{2}\right), 77.3\left(\mathrm{~d},{ }^{4} J_{\mathrm{C}-\mathrm{F}}=2.2 \mathrm{~Hz}, \mathrm{C}\right), 102.5\left(\mathrm{CH}_{2}\right)$, $109.3\left(\mathrm{~d},{ }^{2} J_{\mathrm{C}-\mathrm{F}}=23.2 \mathrm{~Hz}, \mathrm{CH}\right), 115.3\left(\mathrm{~d},{ }^{2} J_{\mathrm{C}-\mathrm{F}}=24.2 \mathrm{~Hz}, \mathrm{CH}\right), 121.9$ $\left(\mathrm{d},{ }^{3} J_{\mathrm{C}-\mathrm{F}}=9.1 \mathrm{~Hz}, \mathrm{CH}\right), 135.3\left(\mathrm{~d},{ }^{4} J_{\mathrm{C}-\mathrm{F}}=2.0 \mathrm{~Hz}, \mathrm{C}\right), 145.3(\mathrm{C}), 153.3$ $\left(\mathrm{d},{ }^{3} J_{\mathrm{C}-\mathrm{F}}=7.1 \mathrm{~Hz}, \mathrm{C}\right), 163.5\left(\mathrm{~d},{ }^{1} J_{\mathrm{C}-\mathrm{F}}=247.5 \mathrm{~Hz}, \mathrm{C}\right)$; MS (EI) $\mathrm{m} / \mathrm{z}$ $178\left(\mathrm{M}^{+}, 37\right), 163$ (100), 133 (33), 88 (12), 61 (17); HRMS (EI) calcd for $\mathrm{C}_{11} \mathrm{H}_{11} \mathrm{FO}\left(\mathrm{M}^{+}\right)$178.0794, found 178.0801. The enantiomeric excess was determined by HPLC analysis with a chiralcel OD-H column $\left(95: 5\right.$ hexane $/{ }^{i} \mathrm{PrOH}$, flow rate of $\left.1.0 \mathrm{~mL} / \mathrm{min}\right): t_{\text {major }}=10.50$ $\min , t_{\text {minor }}=12.92 \mathrm{~min}$; 94:6 er.

(1S)-2,3-Dihydro-6-chloro-1-methyl-3-(methylene)indan-1ol (10f). The reaction was performed according to the general procedure using 1-(2'-bromo-5'-chlorophenyl)ethan-1-one (3f) $(0.10$ g, $0.43 \mathrm{mmol}$ ). The crude mixture was purified by silica gel flash chromatography eluting with $10 \%$ diethyl ether in petroleum ether (40-60) to yield (1S)-2,3-dihydro-6-chloro-1-methyl-3-(methylene)indan-1-ol (10f) (0.044 g, 52\% yield) as a colorless oil: IR (neat) 3372, 2924, 1628, $1458 \mathrm{~cm}^{-1} ;[\alpha]_{\mathrm{D}}{ }^{31}+19.5\left(c 1.2, \mathrm{CHCl}_{3}\right) ;{ }^{1} \mathrm{H}$ NMR $(400$ $\left.\mathrm{MHz}, \mathrm{CD}_{3} \mathrm{OD}\right) \delta 1.48(\mathrm{~s}, 3 \mathrm{H}), 2.89(\mathrm{t}, J=2.0 \mathrm{~Hz}, 1 \mathrm{H}), 5.08(\mathrm{t}, J=2.0$ $\mathrm{Hz}, 1 \mathrm{H}), 5.52(\mathrm{t}, J=2.0 \mathrm{~Hz}, 1 \mathrm{H}), 7.27(\mathrm{dd}, J=8.2,1.9 \mathrm{~Hz}, 1 \mathrm{H}), 7.39$ $(\mathrm{d}, J=1.9 \mathrm{~Hz}, 1 \mathrm{H}), 7.48(\mathrm{~d}, J=8.2 \mathrm{~Hz}, 1 \mathrm{H}) ;{ }^{13} \mathrm{C}$ NMR $(101 \mathrm{MHz}$, $\left.\mathrm{CD}_{3} \mathrm{OD}\right) \delta 27.5\left(\mathrm{CH}_{3}\right), 48.7\left(\mathrm{CH}_{2}\right), 77.4(\mathrm{C}), 103.7\left(\mathrm{CH}_{2}\right), 121.6$ $(\mathrm{CH}), 123.1(\mathrm{CH}), 128.2(\mathrm{CH}), 134.0(\mathrm{C}), 137.9(\mathrm{C}), 145.3(\mathrm{C})$, 152.8 (C); MS (EI) m/z $194\left(\mathrm{M}^{+}, 76\right), 179$ (100), 159 (71), 144 (23), 115 (43), 84 (18); HRMS (EI) calcd for $\mathrm{C}_{11} \mathrm{H}_{11}{ }^{35} \mathrm{ClO}\left(\mathrm{M}^{+}\right)$194.0498, found 194.0491. The enantiomeric excess was determined by HPLC analysis with a chiralcel OD-H column $\left(95: 5\right.$ hexane $/{ }^{i} \mathrm{PrOH}$, flow rate of $1.0 \mathrm{~mL} / \mathrm{min}$ ): $t_{\text {major }}=5.33 \mathrm{~min}, t_{\text {minor }}=5.72 \mathrm{~min}$; $95: 5$ er. 
(1S)-2,3-Dihydro-6-nitro-1-methyl-3-(methylene)indan-1-ol $(10 \mathrm{~g})$. The reaction was performed according to the general procedure using 1-(2'-bromo-5'-nitrophenyl)ethan-1-one (3g) (0.029 g, 0.10 mmol). The crude mixture was purified by silica gel flash chromatography eluting with $30 \%$ diethyl ether in petroleum ether (40-60) to yield (1S)-2,3-dihydro-6-nitro-1-methyl-3-(methylene)indan-1-ol $(10 \mathrm{~g})(0.0090 \mathrm{~g}, 19 \%)$ as a yellow oil: IR (neat) 3372, 2970, 1520, $1342 \mathrm{~cm}^{-1} ;[\alpha]_{\mathrm{D}}{ }^{30}+45.0\left(c 0.5, \mathrm{CHCl}_{3}\right) ;{ }^{1} \mathrm{H}$ NMR (400 $\left.\mathrm{MHz}, \mathrm{CD}_{3} \mathrm{OD}\right) \delta 1.55(\mathrm{~s}, 3 \mathrm{H}), 2.92-3.04(\mathrm{~m}, 2 \mathrm{H}), 5.32(\mathrm{t}, J=2.2 \mathrm{~Hz}$, $1 \mathrm{H}), 5.78(\mathrm{t}, J=2.2 \mathrm{~Hz}, 1 \mathrm{H}), 7.73(\mathrm{~d}, J=8.5 \mathrm{~Hz}, 1 \mathrm{H}), 8.18(\mathrm{dd}, J=$ $8.5,2.2 \mathrm{~Hz}, 1 \mathrm{H}), 8.26(\mathrm{~d}, J=2.2 \mathrm{~Hz}, 1 \mathrm{H}) ;{ }^{13} \mathrm{C} \mathrm{NMR}(126 \mathrm{MHz}$, $\left.\mathrm{CD}_{3} \mathrm{OD}\right) \delta 27.5\left(\mathrm{CH}_{3}\right), 48.5\left(\mathrm{CH}_{2}\right), 77.2(\mathrm{C}), 108.0\left(\mathrm{CH}_{2}\right), 118.5$ $(\mathrm{CH}), 121.2(\mathrm{CH}), 123.5(\mathrm{CH}), 144.8(\mathrm{C}), 145.3(\mathrm{C}), 148.4(\mathrm{C})$, 152.3 (C); MS (ESI) $m / z 228\left(\mathrm{MNa}^{+}, 100\right)$; HRMS (ESI) calcd for $\mathrm{C}_{11} \mathrm{H}_{11} \mathrm{NNaO}_{3}\left(\mathrm{MNa}^{+}\right)$228.0631, found 228.0625. The enantiomeric excess was determined by HPLC analysis with a chiralcel OD-H column $\left(93: 7\right.$ hexane $/{ }^{i} \mathrm{PrOH}$, flow rate of $\left.2.0 \mathrm{~mL} / \mathrm{min}\right): t_{\text {major }}=3.84$ $\min , t_{\text {minor }}=4.18 \mathrm{~min} ; 93.5: 6.5 \mathrm{er}$.

(5S)-5,7-Dimethylcyclopenta[b]pyridin-5-ol $(11 \mathrm{~h})$. The reaction was performed according to the general procedure using 1- $\left(2^{\prime}\right.$ bromo-3'-pyridyl)ethan-1-one (3h) $(0.080 \mathrm{~g}, 0.40 \mathrm{mmol})$. The crude mixture was purified by silica gel flash chromatography eluting with $10 \%$ ethyl acetate in petroleum ether $(40-60)$ to yield (5S)-5,7dimethylcyclopenta[b]pyridin-5-ol $(11 \mathrm{~h})(0.040 \mathrm{~g}, 63 \%)$ as a yellow oil: IR (neat) $3322,2972,1603,1572,1474 \mathrm{~cm}^{-1} ;[\alpha]_{\mathrm{D}}{ }^{31}+24.2(c 0.4$, $\left.\mathrm{CHCl}_{3}\right) ;{ }^{1} \mathrm{H}$ NMR $\left(400 \mathrm{MHz}, \mathrm{CD}_{3} \mathrm{OD}\right) \delta 1.53(\mathrm{~s}, 3 \mathrm{H}), 2.10(\mathrm{~d}, J=$ $1.6 \mathrm{~Hz}, 3 \mathrm{H}), 6.36(\mathrm{q}, J=1.6 \mathrm{~Hz}, 1 \mathrm{H}), 7.19(\mathrm{dd}, J=7.4,5.3 \mathrm{~Hz}, 1 \mathrm{H})$, $7.74(\mathrm{dd}, J=7.4,1.4 \mathrm{~Hz}, 1 \mathrm{H}), 8.30(\mathrm{dd}, J=5.3,1.4 \mathrm{~Hz}, 1 \mathrm{H}) ;{ }^{13} \mathrm{C}$ NMR (101 MHz, CD $\mathrm{OD}) \delta 10.4\left(\mathrm{CH}_{3}\right), 22.8\left(\mathrm{CH}_{3}\right), 77.7(\mathrm{C}), 120.7$ $(\mathrm{CH}), 128.8(\mathrm{CH}), 138.8(\mathrm{C}), 143.1(\mathrm{CH}), 144.5(\mathrm{C}), 147.4(\mathrm{CH})$, 161.9 (C); MS (EI) $m / z 161\left(\mathrm{M}^{+}, 75\right), 146$ (100), 132 (12), 117 (14); HRMS (EI) calcd for $\mathrm{C}_{10} \mathrm{H}_{11} \mathrm{NO}\left(\mathrm{M}^{+}\right) 161.0841$, found 161.0835. The enantiomeric excess was determined by HPLC analysis with a chiralcel OD-H column (95:5 hexane $/{ }^{i} \mathrm{PrOH}$, flow rate of $\left.1.0 \mathrm{~mL} / \mathrm{min}\right): t_{\text {major }}=$ $11.95 \mathrm{~min}, t_{\mathrm{minor}}=18.05 \mathrm{~min} ; 92.5: 7.5 \mathrm{er}$.

(1S)-2,3-Dihydro-1-ethyl-3-(methylene)indan-1-ol (10i). The reaction was performed according to the general procedure using $2^{\prime}$ bromopropiophenone $(3 \mathbf{i})(0.10 \mathrm{~g}, 0.47 \mathrm{mmol})$. The crude mixture was purified by silica gel flash chromatography eluting with $10 \%$ diethyl ether in petroleum ether (40-60) to yield (1S)-2,3-dihydro-1ethyl-3-(methylene)indan-1-ol (10i) $(0.048 \mathrm{~g}, 59 \%)$ as a yellow oil: IR (neat) $3348,2932,1628,1458 \mathrm{~cm}^{-1} ;[\alpha]_{\mathrm{D}}{ }^{31}+8.3\left(c 1.0, \mathrm{CHCl}_{3}\right) ;{ }^{1} \mathrm{H}$ NMR $\left(400 \mathrm{MHz}, \mathrm{CD}_{3} \mathrm{OD}\right) \delta 0.81(\mathrm{t}, J=7.5 \mathrm{~Hz}, 3 \mathrm{H}), 1.76(\mathrm{dq}, J=$ $13.3,7.5 \mathrm{~Hz}, 1 \mathrm{H}), 1.88(\mathrm{dq}, J=13.3,7.5 \mathrm{~Hz}, 1 \mathrm{H}), 2.77(\mathrm{dt}, J=16.4$, $2.1 \mathrm{~Hz}, 1 \mathrm{H}), 2.96(\mathrm{dt}, J=16.4,2.1 \mathrm{~Hz}, 1 \mathrm{H}), 5.04(\mathrm{t}, J=2.1 \mathrm{~Hz}, 1 \mathrm{H})$, $5.49(\mathrm{t}, J=2.1 \mathrm{~Hz}, 1 \mathrm{H}), 7.25-7.32(\mathrm{~m}, 2 \mathrm{H}), 7.35-7.40(\mathrm{~m}, 1 \mathrm{H})$, $7.48-7.53(\mathrm{~m}, 1 \mathrm{H}) ;{ }^{13} \mathrm{C} \mathrm{NMR}\left(126 \mathrm{MHz}, \mathrm{CD}_{3} \mathrm{OD}\right) \delta 7.5\left(\mathrm{CH}_{3}\right), 33.9$ $\left(\mathrm{CH}_{2}\right), 45.7\left(\mathrm{CH}_{2}\right), 80.7(\mathrm{C}), 102.3\left(\mathrm{CH}_{2}\right), 120.0(\mathrm{CH}), 123.3(\mathrm{CH})$, $128.0(\mathrm{CH}), 128.3(\mathrm{CH}), 140.0(\mathrm{C}), 146.8(\mathrm{C}), 149.6$ (C); MS (ESI) $\mathrm{m} / z 197\left(\mathrm{MNa}^{+}, 100\right)$; HRMS (ESI) calcd for $\mathrm{C}_{12} \mathrm{H}_{14} \mathrm{NaO}\left(\mathrm{MNa}^{+}\right)$ 197.0937, found 197.0932. The enantiomeric excess was determined by HPLC analysis with a chiralcel OD-H column (95:5 hexane $/{ }^{i} \mathrm{PrOH}$, flow rate of $1.0 \mathrm{~mL} / \mathrm{min}): t_{\text {major }}=2.38 \mathrm{~min}, t_{\text {minor }}=2.80 \mathrm{~min} ; 94: 6 \mathrm{er}$.

(3S)-2,3-Dihydro-3-hydroxy-3-methyl-1-indanone (14). (3S)2,3-Dihydro-1-methyl-3-(methylene)indan-1-ol ( $S$-10a) (0.300 g, 1.87 $\mathrm{mmol})$ was dissolved in a mixture of dichloromethane $(60 \mathrm{~mL})$ and methanol $(60 \mathrm{~mL})$ and cooled to $-78{ }^{\circ} \mathrm{C}$. The reaction mixture was purged with oxygen, and then ozone was bubbled through until the clear solution turned blue. The excess ozone was purged with oxygen and then with argon. Triphenylphosphine $(1.47 \mathrm{~g}, 5.61 \mathrm{mmol})$ was added portionwise to the mixture while it was being vigorously stirred. The reaction mixture was allowed to return to room temperature over $2 \mathrm{~h}$. The reaction mixture was concentrated in vacuo. The resulting residue was purified by silica gel flash column chromatography eluting with $20 \%$ diethyl ether in petroleum ether (40-60) to give (3S)-2,3dihydro-3-hydroxy-3-methyl-1-indanone (14) $(0.250 \mathrm{~g}, 81 \%)$ as a yellow oil: IR (neat) 3393, 2972, 1701, $1603 \mathrm{~cm}^{-1} ;[\alpha]_{\mathrm{D}}{ }^{31}+96.9$ (c 1.0, $\left.\mathrm{CHCl}_{3}\right) ;{ }^{1} \mathrm{H}$ NMR $\left(400 \mathrm{MHz}, \mathrm{CDCl}_{3}\right) \delta 1.73(\mathrm{~s}, 3 \mathrm{H}), 2.15(\mathrm{~s}, 1 \mathrm{H})$, $2.91(\mathrm{~s}, 2 \mathrm{H}), 7.44-7.53(\mathrm{~m}, 1 \mathrm{H}), 7.68-7.75(\mathrm{~m}, 3 \mathrm{H}) ;{ }^{13} \mathrm{C}$ NMR $(101$ $\left.\mathrm{MHz}, \mathrm{CDCl}_{3}\right) \delta 29.3\left(\mathrm{CH}_{3}\right), 53.8\left(\mathrm{CH}_{2}\right), 74.5(\mathrm{C}), 123.2(\mathrm{CH}), 123.6$
(CH), $129.4(\mathrm{CH}), 135.4(\mathrm{C}), 135.6(\mathrm{CH}), 158.6(\mathrm{C}), 203.2(\mathrm{C})$; MS (EI) $m / z 162\left(\mathrm{M}^{+}, 88\right), 147$ (100), 129 (53), 115 (43), 91 (22), 84 (38), 77 (19); HRMS (EI) calcd for $\mathrm{C}_{10} \mathrm{H}_{10} \mathrm{O}_{2}\left(\mathrm{M}^{+}\right)$162.0681, found 162.0680 .

(1S,3S)-2,3-Dihydro-1-(methyl)indan-1,3-diol (15). To a stirred solution of (3S)-2,3-dihydro-3-hydroxy-3-methyl-1-indanone (14) $(0.020 \mathrm{~g}, 0.12 \mathrm{mmol})$ in 1,2-dichloroethane $(2 \mathrm{~mL})$ were added sodium triacetoxyborohydride $(0.038 \mathrm{~g}, 0.18 \mathrm{mmol})$ and acetic acid $(0.0070 \mathrm{~mL}, 0.12 \mathrm{mmol})$ at room temperature under an argon atmosphere. Sodium triacetoxyborohydride $(0.038 \mathrm{~g}, 0.18 \mathrm{mmol})$ and acetic acid $(0.0070 \mathrm{~mL}, 0.12 \mathrm{mmol})$ were then added after 2 days. The reaction mixture was stirred at room temperature for a total of 6 days and then the reaction quenched with a $1 \mathrm{M}$ sodium hydroxide solution $(2 \mathrm{~mL})$. After phase separation, the aqueous layer was extracted with dichloromethane $(2 \times 5 \mathrm{~mL})$, dried over $\mathrm{MgSO}_{4}$, filtered, and concentrated in vacuo. Purification of the crude material using silica gel flash column chromatography eluting with $5 \%$ methanol in dichloromethane gave (1S,3S)-2,3-dihydro-1-(methyl)indane-1,3-diol (15) $\left(0.015 \mathrm{~g}, 75 \%\right.$ yield) as a white solid: $\mathrm{mp} 97-99{ }^{\circ} \mathrm{C}$; IR (neat) $3331,2967,1092 \mathrm{~cm}^{-1} ;[\alpha]_{\mathrm{D}}{ }^{31}+48.5\left(c 0.6, \mathrm{CHCl}_{3}\right) ;{ }^{1} \mathrm{H}$ NMR (500 $\left.\mathrm{MHz}, \mathrm{CDCl}_{3}\right) \delta 1.70(\mathrm{~s}, 3 \mathrm{H}), 1.76(\mathrm{~s}, 1 \mathrm{H}), 1.90(\mathrm{~s}, 1 \mathrm{H}), 2.10(\mathrm{dd}, J=$ 13.5, $5.0 \mathrm{~Hz}, 1 \mathrm{H}), 2.63(\mathrm{dd}, J=13.5,6.5 \mathrm{~Hz}, 1 \mathrm{H}), 5.41$ (br t, $J=6.0$ $\mathrm{Hz}, 1 \mathrm{H}), 7.33-7.45(\mathrm{~m}, 4 \mathrm{H}) ;{ }^{13} \mathrm{C}$ NMR $\left(126 \mathrm{MHz} \mathrm{CDCl}_{3}\right) \delta 28.7$ $\left(\mathrm{CH}_{3}\right), 52.6\left(\mathrm{CH}_{2}\right), 73.6(\mathrm{CH}), 79.4(\mathrm{C}), 122.3(\mathrm{CH}), 124.5(\mathrm{CH})$, $129.1(\mathrm{CH}), 129.1(\mathrm{CH}), 144.3(\mathrm{C}), 147.4(\mathrm{C})$; MS (ESI) $\mathrm{m} / z 187$ $\left(\mathrm{MNa}^{+}, 98\right)$; HRMS (ESI) calcd for $\mathrm{C}_{10} \mathrm{H}_{12} \mathrm{NaO}_{2}\left(\mathrm{MNa}^{+}\right)$187.0730, found 187.0730.

General Procedure for the Reductive Amination Reactions. To a solution of (3S)-2,3-dihydro-3-hydroxy-3-methyl-1-indanone (14) (1 equiv) in 1,2-dichloroethane $(2 \mathrm{~mL} / \mathrm{mmol})$ were added the amine (1.1-1.5 equiv) and sodium triacetoxyborohydride (1.4-1.5 equiv) at room temperature under an argon atmosphere. The same amounts of amine and hydride source were added after 2 days. The reaction mixture was stirred at room temperature for $\leq 6$ days and then the reaction quenched with a $1 \mathrm{M}$ sodium hydroxide solution $(2 \mathrm{~mL} /$ $\mathrm{mmol})$. After phase separation, the aqueous layer was extracted with dichloromethane $(2 \times 5 \mathrm{~mL} / \mathrm{mmol})$, dried $\left(\mathrm{MgSO}_{4}\right)$, filtered, and concentrated in vacuo. Purification using silica gel flash column chromatography eluting with methanol in dichloromethane gave the 3amino-substituted 2,3-dihydro-1-(methyl)indan-1-ols.

(1S,3S)-3-Benzylamino-2,3-dihydro-1-(methyl)indan-1-ol (16a). The reaction was performed according to the general procedure using (3S)-2,3-dihydro-3-hydroxy-3-methyl-1-indanone (14) (0.032 g, $0.20 \mathrm{mmol})$ and benzylamine $(0.024 \mathrm{~mL}, 0.22 \mathrm{mmol})$. The reaction mixture was stirred at room temperature for 3 days. Purification of the crude material using silica gel flash column chromatography eluting with $5 \%$ methanol in dichloromethane gave $(1 S, 3 S)$-3-benzylamino2,3-dihydro-1-(methyl)indan-1-ol (16a) $(0.042 \mathrm{~g}, 84 \%)$ as a yellow oil: IR (neat) 3312, 2965, $1452 \mathrm{~cm}^{-1}$; $[\alpha]_{\mathrm{D}}{ }^{31}+22.9\left(\right.$ c $\left.1.0, \mathrm{CHCl}_{3}\right) ;{ }^{1} \mathrm{H}$ $\operatorname{NMR}\left(500 \mathrm{MHz}, \mathrm{CDCl}_{3}\right) \delta 1.60-1.77(\mathrm{~m}, 5 \mathrm{H}), 1.98(\mathrm{dd}, J=13.2,6.5$ $\mathrm{Hz}, 1 \mathrm{H}), 2.59(\mathrm{dd} J=13.2,6.5 \mathrm{~Hz}, 1 \mathrm{H}), 3.88(\mathrm{~d}, J=13.0 \mathrm{~Hz}, 1 \mathrm{H})$, $3.93(\mathrm{~d}, J=13.0 \mathrm{~Hz}, 1 \mathrm{H}), 4.48(\mathrm{t}, J=6.5 \mathrm{~Hz}, 1 \mathrm{H}), 7.22-7.44(\mathrm{~m}$, $9 \mathrm{H}) ;{ }^{13} \mathrm{C}$ NMR $\left(126 \mathrm{MHz}, \mathrm{CDCl}_{3}\right) \delta 28.1\left(\mathrm{CH}_{3}\right), 50.4\left(\mathrm{CH}_{2}\right), 51.7$ $\left(\mathrm{CH}_{2}\right), 59.8(\mathrm{CH}), 79.4(\mathrm{C}), 122.3(\mathrm{CH}), 124.5(\mathrm{CH}), 127.0(\mathrm{CH})$, $128.2(2 \times \mathrm{CH}), 128.2(\mathrm{CH}), 128.5(2 \times \mathrm{CH}), 128.7(\mathrm{CH}), 140.5$ (C), 145.0 (C), 147.4 (C); MS (ESI) $m / z 254\left(\mathrm{MH}^{+}, 100\right)$; HRMS (ESI) calcd for $\mathrm{C}_{17} \mathrm{H}_{20} \mathrm{NO}\left(\mathrm{MH}^{+}\right)$254.1539, found 254.1534.

(1S,3S)-2,3-Dihydro-3-(4'-methoxybenzyl)amino-1-(methyl)indan-1-ol (16b). The reaction was performed according to the general procedure using (3S)-2,3-dihydro-3-hydroxy-3-methyl-1-indanone $(14)(0.020 \mathrm{~g}, 0.12 \mathrm{mmol})$ and 4-methoxybenzylamine $(0.018$ $\mathrm{mL}, 0.014 \mathrm{mmol})$. The reaction mixture was stirred at room temperature for 6 days. Purification of the crude material using silica gel flash column chromatography eluting with $5 \%$ methanol in dichloromethane gave (1S,3S)-2,3-dihydro-3-(4'-methoxybenzyl)amino-1-(methyl)indan-1-ol $(\mathbf{1 6 b})(0.029 \mathrm{~g}, 84 \%)$ as a yellow oil: IR (neat) 3360, 2961, 1611, $1512 \mathrm{~cm}^{-1} ;[\alpha]_{\mathrm{D}}^{31}+19.1$ (c 1.0, $\left.\mathrm{CHCl}_{3}\right)$; ${ }^{1} \mathrm{H}$ NMR $\left(400 \mathrm{MHz}, \mathrm{CDCl}_{3}\right) \delta 1.50-1.77(\mathrm{~m}, 5 \mathrm{H}), 1.99(\mathrm{dd}, J=13.3$, $6.5 \mathrm{~Hz}, 1 \mathrm{H}), 2.60(\mathrm{dd}, J=13.3,6.5 \mathrm{~Hz}, 1 \mathrm{H}), 3.80(\mathrm{~s}, 3 \mathrm{H}), 3.83(\mathrm{~d}, J=$ $12.8 \mathrm{~Hz}, 1 \mathrm{H}), 3.88(\mathrm{~d}, J=12.8 \mathrm{~Hz}, 1 \mathrm{H}), 4.49(\mathrm{t}, J=6.5 \mathrm{~Hz}, 1 \mathrm{H})$, 
6.83-6.91 (m, 2H), 7.27-7.43 (m, 6H); ${ }^{13} \mathrm{C}$ NMR (101 MHz, $\left.\mathrm{CDCl}_{3}\right) \delta 28.1\left(\mathrm{CH}_{3}\right), 50.4\left(\mathrm{CH}_{2}\right), 51.2\left(\mathrm{CH}_{2}\right), 55.3\left(\mathrm{CH}_{3}\right), 59.7$ $(\mathrm{CH}), 79.4(\mathrm{C}), 113.8(2 \times \mathrm{CH}), 122.2(\mathrm{CH}), 124.4(\mathrm{CH}), 128.2$ (CH), $128.7(\mathrm{CH}), 129.3(2 \times \mathrm{CH}), 132.6(\mathrm{C}), 145.1(\mathrm{C}), 147.3(\mathrm{C})$, 158.7 (C); MS (ESI) $\mathrm{m} / z 284\left(\mathrm{MH}^{+}, 100\right)$; HRMS (ESI) calcd for $\mathrm{C}_{18} \mathrm{H}_{22} \mathrm{NO}_{2}\left(\mathrm{MH}^{+}\right)$284.1645, found 284.1640.

(1S,3S)-3-Allylamino-2,3-dihydro-1-(methyl)indan-1-ol (16c). The reaction was performed according to the general procedure using (3S)-2,3-dihydro-3-hydroxy-3-methyl-1-indanone (14) (0.020 g, 0.12 $\mathrm{mmol})$ and allylamine $(0.013 \mathrm{~mL}, 0.18 \mathrm{mmol})$. The reaction mixture was stirred at room temperature for 6 days. Purification of the crude material using silica gel flash column chromatography eluting with $5 \%$ methanol in dichloromethane gave (1S,3S)-3-allylamino-2,3-dihydro1-(methyl)indan-1-ol (16c) $(0.019 \mathrm{~g}, 78 \%)$ as a yellow oil: IR (neat) $3329,2967,1452 \mathrm{~cm}^{-1} ;[\alpha]_{\mathrm{D}}{ }^{31}+30.3\left(c 0.8, \mathrm{CHCl}_{3}\right)$; ${ }^{1} \mathrm{H}$ NMR (500 $\left.\mathrm{MHz}, \mathrm{CDCl}_{3}\right) \delta 1.55-1.85(\mathrm{~m}, 5 \mathrm{H}), 1.93(\mathrm{dd}, J=13.3,6.7 \mathrm{~Hz}, 1 \mathrm{H})$, $2.60(\mathrm{dd}, J=13.3,6.7 \mathrm{~Hz}, 1 \mathrm{H}), 3.37(\mathrm{dd}, J=14.0,6.0 \mathrm{~Hz}, 1 \mathrm{H}), 3.42$ $(\mathrm{dd}, J=14.0,6.0 \mathrm{~Hz}, 1 \mathrm{H}), 4.49(\mathrm{t}, J=6.7 \mathrm{~Hz}, 1 \mathrm{H}), 5.13(\mathrm{dd}, J=10.5$, $1.5 \mathrm{~Hz}, 1 \mathrm{H}), 5.25(\mathrm{dd}, J=17.0,1.5 \mathrm{~Hz}, 1 \mathrm{H}), 5.98(\mathrm{ddt}, J=17.0,10.5$, $6.0 \mathrm{~Hz}, 1 \mathrm{H}), 7.28-7.44(\mathrm{~m}, 4 \mathrm{H}) ;{ }^{13} \mathrm{C} \mathrm{NMR}\left(126 \mathrm{MHz} \mathrm{CDCl}_{3}\right) \delta$ $28.0\left(\mathrm{CH}_{3}\right), 50.2\left(\mathrm{CH}_{2}\right), 50.4\left(\mathrm{CH}_{2}\right), 59.7(\mathrm{CH}), 79.3(\mathrm{C}), 116.2$ $\left(\mathrm{CH}_{2}\right), 122.3(\mathrm{CH}), 124.4(\mathrm{CH}), 128.3(\mathrm{CH}), 128.8(\mathrm{CH}), 136.7$ (CH), 144.8 (C), 147.3 (C); MS (ESI) $m / z 204\left(\mathrm{MH}^{+}, 100\right)$; HRMS (ESI) calcd for $\mathrm{C}_{13} \mathrm{H}_{18} \mathrm{NO}\left(\mathrm{MH}^{+}\right)$204.1383, found 204.1382.

(1S,3S)-2,3-Dihydro-1-methyl-3-(morpholino)indan-1-ol (16d). The reaction was performed according to the general procedure using (3S)-2,3-dihydro-3-hydroxy-3-methyl-1-indanone (14) (0.020 g, $0.12 \mathrm{mmol})$ and morpholine $(0.016 \mathrm{~mL}, 0.18 \mathrm{mmol})$. The reaction mixture was stirred at rt for 6 days. Purification of the crude material using silica gel flash column chromatography eluting with $8 \%$ methanol in dichloromethane gave (1S,3S)-2,3-dihydro-1-methyl-3(morpholino)indan-1-ol (16d) (0.022 g, 79\%) as a light brown solid: mp 84-86 ${ }^{\circ} \mathrm{C}$; IR (neat) 3410, 2961, $1452 \mathrm{~cm}^{-1} ;[\alpha]_{\mathrm{D}}^{31}+74.8(c 0.9$, $\left.\mathrm{CHCl}_{3}\right) ;{ }^{1} \mathrm{H}$ NMR $\left(400 \mathrm{MHz}, \mathrm{CDCl}_{3}\right) \delta 1.65-1.77(\mathrm{~m}, 4 \mathrm{H}), 2.20(\mathrm{~d}$, $J=7.2 \mathrm{~Hz}, 2 \mathrm{H}), 2.48(\mathrm{dt}, J=11.2,4.4 \mathrm{~Hz}, 2 \mathrm{H}), 2.53(\mathrm{dt}, J=11.2,4.4$ $\mathrm{Hz}, 2 \mathrm{H}), 3.71(\mathrm{t}, J=4.4 \mathrm{~Hz}, 4 \mathrm{H}), 4.54(\mathrm{t}, J=7.2 \mathrm{~Hz}, 1 \mathrm{H}), 7.27-7.42$ (m, 4H); ${ }^{13} \mathrm{C}$ NMR $\left(101 \mathrm{MHz}, \mathrm{CDCl}_{3}\right) \delta 27.5\left(\mathrm{CH}_{3}\right), 40.5\left(\mathrm{CH}_{2}\right)$, 49.2 $\left(2 \times \mathrm{CH}_{2}\right), 67.2(\mathrm{CH}), 67.4\left(2 \times \mathrm{CH}_{2}\right), 79.1(\mathrm{C}), 122.2(\mathrm{CH})$, $125.5(\mathrm{CH}), 128.3(\mathrm{CH}), 128.5(\mathrm{CH}), 142.5(\mathrm{C}), 147.6(\mathrm{C})$; MS (ESI) $m / z 234\left(\mathrm{MH}^{+}, 100\right)$; HRMS (ESI) calcd for $\mathrm{C}_{14} \mathrm{H}_{20} \mathrm{NO}_{2}$ $\left(\mathrm{MH}^{+}\right)$234.1489, found 234.1490.

\section{ASSOCIATED CONTENT}

\section{S Supporting Information}

The Supporting Information is available free of charge on the ACS Publications website at DOI: 10.1021/acs.joc.7b02287.

HPLC traces for all indanols and, ${ }^{1} \mathrm{H}$ and ${ }^{13} \mathrm{C}$ NMR spectra for all compounds (PDF)

X-ray data for compound 15 (CIF)

$\mathrm{X}$-ray data for compound $16 \mathrm{~d}$ (CIF)

\section{AUTHOR INFORMATION}

\section{Corresponding Author}

*E-mail: andrew.sutherland@glasgow.ac.uk

ORCID

Andrew Sutherland: 0000-0001-7907-5766

Notes

The authors declare no competing financial interest.

\section{ACKNOWLEDGMENTS}

Financial support from the University of Glasgow (studentship to R.J.F.) and EPSRC (studentship to E.D.D.C., EP/P505534/ 1) is gratefully acknowledged.

\section{REFERENCES}

(1) Hong, B.-c.; Sarshar, S. Org. Prep. Proced. Int. 1999, 31, 1.
(2) Loh, T.-P.; Hu, Q.-Y. Org. Lett. 2001, 3, 279.

(3) Froimowitz, M.; Wu, K.-M.; Moussa, A.; Haidar, R. M.; Jurayj, J.; George, C.; Gardner, E. L. J. Med. Chem. 2000, 43, 4981.

(4) Kim, S.-H.; Kwon, S. H.; Park, S.-H.; Lee, J. K.; Bang, H.-S.; Nam, S.-J.; Kwon, H. C.; Shin, J.; Oh, D.-C. Org. Lett. 2013, 15, 1834.

(5) For example, see: (a) Kündig, E. P.; Ratni, H.; Crousse, B.; Bernardinelli, G. J. Org. Chem. 2001, 66, 1852. (b) Kesavan, S.; Panek, J. S.; Porco, J. A., Jr Org. Lett. 2007, 9, 5203. (c) Mirabdolbaghi, R; Dudding, T. Tetrahedron 2012, 68, 1988.

(6) (a) Cvengroš, J.; Schütte, J.; Schlörer, N.; Neudörfl, J.; Schmalz, H.-G. Angew. Chem., Int. Ed. 2009, 48, 6148. (b) Schütte, J.; Ye, S.; Schmalz, H.-G. Synlett 2011, 2011, 2725.

(7) Tada, A.; Tokoro, Y.; Fukuzawa, S.-i. J. Org. Chem. 2014, 79, 7905.

(8) Calder, E. D. D.; Sutherland, A. Org. Lett. 2015, 17, 2514.

(9) For nongeneral examples, see: (a) Sasaoka, M.; Hart, H. J. Org. Chem. 1979, 44, 368. (b) Yu, X.; Lu, X. Org. Lett. 2009, 11, 4366.

(10) Mahendar, L.; Satyanarayana, G. J. Org. Chem. 2014, 79, 2059.

(11) Gai, X.; Grigg, R.; Collard, S.; Muir, J. E. Chem. Commun. 2000, 1765.

(12) Oestreich, M.; Sempere-Culler, F.; Machotta, A. B. Angew. Chem., Int. Ed. 2005, 44, 149.

(13) The use of a desymmetrizing intramolecular Heck reaction for the racemic synthesis of a 1-allyl-3-methyleneindan-1-ol has also been reported: Coogan, M. P.; Pottenger, M. J. J. Organomet. Chem. 2005, 690, 1409.

(14) For a review, see: Yus, M.; González-Gómez, J. C.; Foubelo, F. Chem. Rev. 2011, 111, 7774.

(15) Using allylborane reagents: (a) Wada, R.; Oisaki, K.; Kanai, M.; Shibasaki, M. J. Am. Chem. Soc. 2004, 126, 8910. (b) Lou, S.; Moquist, P. N.; Schaus, S. E. J. Am. Chem. Soc. 2006, 128, 12660. (c) Barnett, D. S.; Moquist, P. N.; Schaus, S. E. Angew. Chem., Int. Ed. 2009, 48, 8679. (d) Shi, S.-L.; Xu, L.-W.; Oisaki, K.; Kanai, M.; Shibasaki, M. J. Am. Chem. Soc. 2010, 132, 6638. (e) Robbins, D. W.; Lee, K.; Silverio, D. L.; Volkov, A.; Torker, S.; Hoveyda, A. H. Angew. Chem., Int. Ed. 2016, 55, 9610.

(16) Using a Sakurai-Hosomi allylation: Wadamoto, M.; Yamamoto, H. J. Am. Chem. Soc. 2005, 127, 14556.

(17) Using a Barbier-type allylation: Haddad, T. D.; Hirayama, L. C.; Taynton, P.; Singaram, B. Tetrahedron Lett. 2008, 49, 508.

(18) Using a Nozaki-Hiyama-Kishi-type allylation: (a) Miller, J. J.; Sigman, M. S. J. Am. Chem. Soc. 2007, 129, 2752. (b) Huang, X.-R.; Chen, C.; Lee, G.-H.; Peng, S.-M. Adv. Synth. Catal. 2009, 351, 3089.

(19) Using allylstannane reagents: (a) Kii, S.; Maruoka, K. Chirality 2003, 15, 68. (b) Kim, J. G.; Waltz, K. M.; Garcia, I. F.; Kwiatkowski, D.; Walsh, P. J. J. Am. Chem. Soc. 2004, 126, 12580.

(20) Jain, P.; Antilla, J. C. J. Am. Chem. Soc. 2010, 132, 11884.

(21) Isomer S-11a is likely formed at the end of the Mizoroki-Heck reaction by isomerization of 3-methyleneindan-1-ol $S$-10a while complexed to the Pd catalyst. Similar results were observed by Mahendar and Satyanarayana (see ref 10)

(22) Cairns, A. G.; Senn, H. M.; Murphy, M. P.; Hartley, R. C. Chem. - Eur. J. 2014, 20, 3742.

(23) For X-ray crystallography data, see the Supporting Information: ORTEP drawings of 15 and 16d and cif files CCDC 1568110 and 1568111. These data can be obtained free of charge from The Cambridge Crystallographic Data Centre via www.ccdc.cambridge/ac/ uk/data_request/cif.

(24) Thomé, I.; Besson, C.; Kleine, T.; Bolm, C. Angew. Chem., Int. Ed. 2013, 52, 7509 .

(25) Matsuda, T.; Shigeno, M.; Makino, M.; Murakami, M. Org. Lett. 2006, 8, 3379.

(26) Kou, X.; Li, Y.; Wu, L.; Zhang, X.; Yang, G.; Zhang, W. Org. Lett. 2015, 17, 5566.

(27) Mckinnon, D. M.; Abouzeid, A. J. Heterocycl. Chem. 1991, 28, 347.

(28) Krishna, J.; Reddy, A. G. K.; Satyanarayana, G. Tetrahedron Lett. 2014, 55, 861. 